\title{
CONVERGENCE RATE OF A FINITE VOLUME SCHEME FOR A TWO DIMENSIONAL CONVECTION-DIFFUSION PROBLEM
}

\author{
Yves Coudière $^{1}$, Jean-Paul Vila ${ }^{1}$ and Philippe Villedieu ${ }^{2}$
}

\begin{abstract}
In this paper, a class of cell centered finite volume schemes, on general unstructured meshes, for a linear convection-diffusion problem, is studied. The convection and the diffusion are respectively approximated by means of an upwind scheme and the so called diamond cell method [4]. Our main result is an error estimate of order $h$, assuming only the $W^{2, p}$ (for $p>2$ ) regularity of the continuous solution, on a mesh of quadrangles. The proof is based on an extension of the ideas developed in [12]. Some new difficulties arise here, due to the weak regularity of the solution, and the necessity to approximate the entire gradient, and not only its normal component, as in [12].

Résumé. Dans cet article, on étudie une classe de schémas volumes finis sur des maillages stucturés généraux, pour un problème linéaire de convection diffusion. La convection est approchée par un schéma décentré amont, et la diffusion par un schéma dit "des cellules diamants" [4]. On démontre une estimation d'erreur d'ordre $h$ pour une solution continue dans $W^{2, p}(p>2)$, sur des maillages de quadrangles. La démonstration est une généralisation des idées de [12]. Les nouvelles difficultés sont la régularité plus faible de la solution exacte et la nécessité de construire une approximation du gradient et pas seulement de sa composante normale aux interfaces.
\end{abstract}

AMS Subject Classification. 65C20, 65N12, 65N15, 76R50, 45L10.

Received: September 24, 1996. Revised: November 14, 1997 and May 4, 1998.

\section{INTRODUCTION}

The aim of this paper is to provide a general framework to analyse the convergence of a particular class of cell centered finite volume schemes (the so called diamond cell method $[4,5,14,22]$ ), on structured or unstructured meshes, for the following linear convection-diffusion equation:

$$
\begin{cases}-\operatorname{div}(A \nabla u)+\operatorname{div}(\mathbf{v} u)=f & \text { in } \Omega \\ u_{\mid \Gamma}=g & \text { in } \Gamma .\end{cases}
$$

It is supposed

- $\Omega$ to be an open convex bounded polygonal set of $\mathbb{R}^{2}$, of boundary $\Gamma$,

- $A$ to be a symmetric definite positive matrix with coefficients in $C^{2}(\bar{\Omega})$,

Keywords and phrases. Finite volumes, convection diffusion, convergence rate, unstructured meshes.

1 Mathematiques pour l'Industrie et la Physique, UMR CNRS-UPS 5640, INSA, Domaine Scientifique de Rangueil, 31077

Toulouse Cedex 4, France. e-mail: Coudiere@gmm.insa-tlse.fr

2 ONERA, Centre de Toulouse, 2 avenue Edouard Belin, 31055 Toulouse Cedex, France. 
- $\mathbf{v}$ to be a vector in $\left(C^{1}(\bar{\Omega})\right)^{2}$ such that $\operatorname{div} \mathbf{v} \geq 0$,

- $g$ to be in $V(\Gamma)=\gamma_{0}\left(W^{2, p}(\Omega)\right)$, the space of the traces on $\Gamma$ of $W^{2, p}(\Omega)$.

For sake of simplicity, we consider the case of a Dirichlet boundary condition, although convergence may be studied similarly for other boundary conditions (Neumann type for instance [6]).

Assuming $f$ to be in $L^{p}(\Omega)$, the solution $u$ of $(1)$ is known to be in $W^{2, p}(\Omega)$, under the condition $2<p<p_{0}$ in the $2 \mathrm{D}$ case (where $p_{0}$ depends on the least angle of the convex polygon $\Omega[7]$ ).

In the first part, the family of meshes $\left(T_{h}\right)_{h>0}$ considered for the discretization is general, including any kind of convex polygonal cells, but satisfying some classical hypotheses of regularity.

There exists $\alpha, \beta, \gamma>0$, such that for $h=\max _{K \in T_{h}} \delta(K)$ we have:

$$
\begin{aligned}
& \forall K \in T_{h}, \quad \alpha h^{2} \leq m(K), \quad \text { Card } \partial K \leq \gamma \\
& \forall e \in \partial K, \quad \beta h \leq m(e) .
\end{aligned}
$$

$\delta(K), m(K), \partial K$ and $m(e)$ are, respectively, the diameter, the measure and the set of the edges $e$ of the polygon $K$, and the measure of such an edge $e$.

Based on the integral form of (1) on the grid elements, the finite volume discretization requires to approximate the fluxes of $\mathbf{v} u$ and $A \nabla u$ (resp. flux of convection and flux of diffusion) on the interfaces of the cells. In our case, the simplest upwind numerical flux is chosen for the convection part. Afterwards, the main point is the approximation of the diffusion part. The only way to deal with general meshes and diffusion matrices is to build an approximation of the entire gradient on each edge of the mesh. There are in the literature prevalently two separate classes of reconstruction, known as Green-Gauss type (tested in $[2,4,5,14,22]$ ), and polynomial Lagrangian interpolation (examined in $[8,15]$ ). Those two methods both take into account more cells than the ones only neighboring an edge. The diamond cell method is of Green-Gauss type and will be described and analysed in this paper. The gradient along an edge is approximated by using all the cells which share a common node with the edge.

The diffusion may also be approached by using cell vertex methods (see for instance [11,16, 17]). They require to discretize the diffusion and the convection on two distinct meshes. But the cell centered methods are generally simpler, and the most widely used, and in consequence focused on in this paper.

A review of the results of convergence of finite volume cell centered schemes for convection-diffusion problems reveals the existence of two techniques of demonstration. The mixed finite element method is used in [3,18-20] to reach an error estimate on meshes of quadrangles and of triangles, but with some restrictions due to the principles of finite element methods (conform meshes made either of triangles or of quadrangles). They also obtain a few results for a three dimensional problem. The second method is due to Herbin who proved by means of finite volume techniques an error estimate for the VF4 scheme [12], defined for a simpler problem on conform meshes of triangles. It is remarkable that this scheme (as well as her results) can also be obtained by using the first method, or by using the diamond cell method of discretization described below. It has been extended to more general problems with a matrix of diffusion and to a wider class of meshes including polygonal cells (Voronoï meshes for instance, [13]) in case of an exact solution being in $C^{2}(\Omega)$ and lately in case of a solution being in $H^{2}$, but for a mesh of rectangles [21].

Our proof is inspired by the ideas of Herbin, but we point out the generality of the equation and of the meshes on which we carry them out. Apart from the general framework described here (Th. 5.1), our actual result is the $h$ convergence rate of the diamond cell approximation of (1) on some regular meshes of quadrangles, in case that the exact solution is in $W^{2, p}(\Omega)$ (Th. 6.1).

Finally, let us mention that the diamond cell method may also apply in the three dimensional case [6]. Our general theorem (weak consistency and coercivity implying convergence) remains true.

This paper is organized as follows. In the next section, we define the numerical scheme and precise the notations. In Section 3, there is given a condition on the gradient reconstruction procedure, sufficient to ensure the weak consistency of the scheme. In Section 4 we define a notion of coercivity; and then prove a fundamental 
result in Section 5: weak consistency and coercivity imply convergence. In Section 6 the framework of Sections 3 to 5 is applied and leads to an error estimate in $h$ for the diamond cell scheme on regular meshes of quadrangles.

\section{The Finite VOlume SCHEME}

\subsection{Notations}

Equation (1) has the following integral form on any $K$ in $T_{h}$.

$$
\sum_{e \in \partial K} \int_{e} u\left(\left(\mathbf{v} \cdot \mathbf{n}_{K e}\right)-(A \nabla u) \cdot \mathbf{n}_{K e}\right) \mathrm{d} s=\int_{K} f \mathrm{~d} x .
$$

$T_{h}$ is the mesh while for each edge $e$ of $\partial K, \mathbf{n}_{K e}$ denotes the normal to $e$ outward to $K$. Equation (3) can also be written as

$$
\sum_{e \in \partial K} s_{K e}\left(\phi_{e}^{C}(u)-\phi_{e}^{D}(u)\right) m(e)=m(K) f_{K}
$$

with

$$
\phi_{e}^{C}(u)=\frac{1}{m(e)} \int_{e} u\left(\mathbf{v} \cdot \mathbf{n}_{e}\right) \mathrm{d} s, \quad \phi_{e}^{D}(u)=\frac{1}{m(e)} \int_{e}(A \nabla u) \cdot \mathbf{n}_{e} \mathrm{~d} s .
$$

$f_{K}$ is the mean value of $f$ on $K$ and $\mathbf{n}_{e}$ is the normal to $e$ such that $\mathbf{v}_{e} \cdot \mathbf{n}_{e}>0$ ( $\mathbf{v}_{e}$ is the mean value of $\mathbf{v}$ along $e$ ) and then $s_{K e}=\mathbf{n}_{K e} \cdot \mathbf{n}_{e}$.

We are looking for an approximation $u_{h}$ constant and of value $u_{K}$ on each cell $K\left(u_{h}=\left(u_{K}\right)_{K \in T_{h}}\right)$ which should represent the mean value of $u$ on $K$. The finite volume discretization proceeds by approximating the fluxes of convection $\phi_{e}^{C}(u)$ and of diffusion $\phi_{e}^{D}(u)$ by some numerical fluxes respectively noted $\phi_{e}^{C}\left(u_{h}\right)$ and $\phi_{e}^{D}\left(u_{h}\right)$.

We shall use capital letters such as $K, N, S$ to denote the elements of the mesh such as the cells or the vertices, while point coordinates are denoted by small letters, such as $x, y$.

In addition to the previous ones, we shall also use the following notations to write the scheme (see Fig. 2.1).

- Concerning a cell $K$

$-x_{K}$ is the centre of gravity of $K$.

- Concerning the edges

$-S_{h}$ is the set of all the edges $e$.

$-S_{h}^{\star}$ is the set of the edges interior to $\Omega$ (such that $e \Varangle \Gamma$ ).

$-\Gamma_{h}$ is the set of the edges in $\Gamma$ (such that $e \subset \Gamma$ ).

- $\mathbf{t}_{e}$ is a unit vector parallel to $e$ such that $\left(\mathbf{n}_{e}, \mathbf{t}_{e}\right)$ is a local direct basis (we recall that $\mathbf{n}_{e}$ is the unit vector normal to $e$ in the direction of the mean velocity of convection $\mathbf{v}_{e}$ ).

- An edge $e$ is an open segment of endpoints $N$ and $S$ (which coordinates are $x_{N}$ and $x_{S}$ ) such that $\left(x_{N}-x_{S}\right) \cdot \mathbf{t}_{e}>0$.

- Around an edge $e, E$ and $W$ denote the downstream and upstream cells with respect to the convection direction $\mathbf{v}_{e}$, one of which $\left(E\right.$ or $W$ ) may reduce to the edge itself (in case $e \in \Gamma_{h}$ ) and then $x_{E \text { or } W}$ is the midpoint of $e$.

- $\chi_{e}$ is the polygon of vertices $x_{N}, x_{E}, x_{S}, x_{W}$ and is called co-volume associated to $e$.

- Concerning the vertices

$-N_{h}$ is the set of all the vertices $P$ of the mesh.

$-N_{h}^{\star}$ is the set of the vertices $P$ interior to $\Omega$ (such that $P \notin \Gamma$ ).

- For $P \in N_{h}, \mathcal{V}_{P}$ is the set of the cells $K \in T_{h}$ which share $P$ as a common node.

Throughout the paper, we shall use $C$ to denote a generic positive constant which is independent of any mesh used (and depends only on $A, \mathbf{v}$ and $\Omega$ ). 


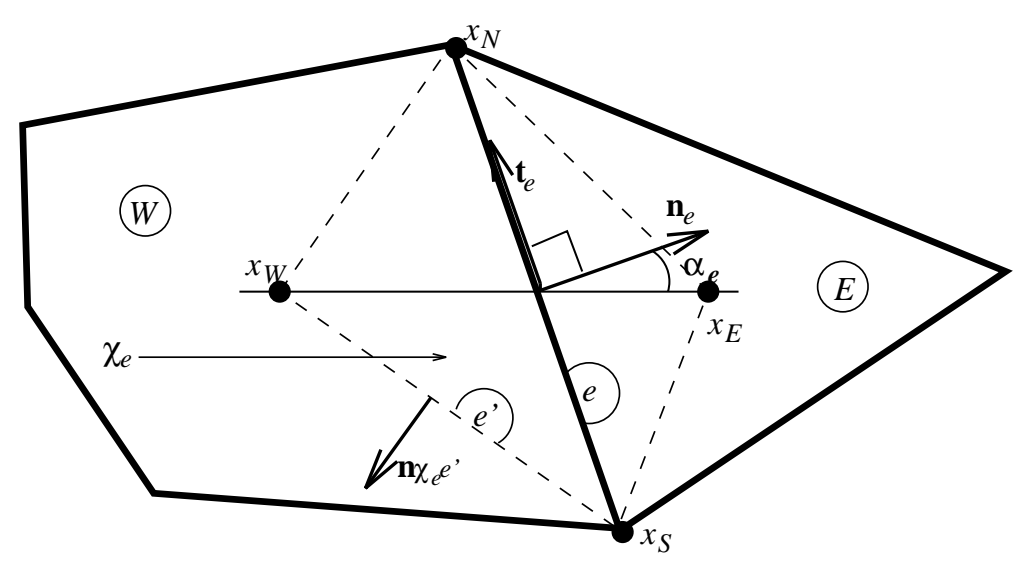

FIGURE 2.1.

\subsection{The numerical flux of convection}

It is given by the classical upwind approximation:

$$
\forall e \in S_{h}, \quad \phi_{e}^{C}\left(u_{h}\right)=u_{W}\left(\mathbf{v}_{e} \cdot \mathbf{n}_{e}\right) .
$$

If $e \in \Gamma_{h}$ and $\mathbf{n}_{e}=-\mathbf{n}_{\text {ext }}$ (i.e. $W$ is reduced to the edge), then we take

$$
u_{W}=g\left(x_{W}\right)
$$

$g\left(x_{W}\right)$ makes sense because of the continuous imbedding of $V(\Gamma)$ in $C^{0}(\Gamma)$ (see [1]).

\subsection{The numerical flux of diffusion}

The essence of the flux of diffusion $\left(\Phi_{e}^{D}(u)\right)$ approximation is the reconstruction of the gradient $\nabla u$ from the cell values $u_{K}$. The exact solution verifies:

$$
\frac{1}{m\left(\chi_{e}\right)} \int_{\chi_{e}} \nabla u \mathrm{~d} x=\frac{1}{m\left(\chi_{e}\right)} \int_{\partial \chi_{e}} u \mathbf{n}_{\text {ext }} \mathrm{d} s .
$$

A formal discretization of (6) leads to the following natural approximation of the gradient on $e$

$$
\mathbf{p}_{e}=\frac{1}{m\left(\chi_{e}\right)} \sum_{e^{\prime} \in \partial \chi_{e}} \frac{1}{2}\left(u_{N_{1}\left(e^{\prime}\right)}+u_{N_{2}\left(e^{\prime}\right)}\right) m\left(e^{\prime}\right) \mathbf{n}_{\chi_{e} e^{\prime}},
$$

where $\chi_{e}$ is the co-volume associated to $e$, and noting $N_{1}\left(e^{\prime}\right)$ and $N_{2}\left(e^{\prime}\right)$ the endpoints of an edge $e^{\prime}$ of $\partial \chi_{e}$ and $\mathbf{n}_{\chi_{e} e^{\prime}}$ its outward unit normal. This kind of reconstruction is commonly called Green-Gauss type (see $[4,5]$ and the references therein). The specific name (diamond-cell) of our method is due to the choice of $\chi_{e}$ as a diamond-shaped polygon. The values at the centres $x_{E}$ and $x_{W}$ are $u_{E}$ and $u_{W}$, while the values at the vertices $x_{N}$ and $x_{S}$ are linearly interpolated from $u_{h}$ (or issued of the boundary condition if necessary) and noted $u_{N}$ and $u_{S}$ :

$$
\begin{aligned}
\text { for } P \in N_{h}^{\star}, \quad u_{P} & =\sum_{K \in \mathcal{V}_{P}} y_{K}(P) u_{K}, \\
\text { for } P \in N_{h} \cap \Gamma, \quad u_{P} & =g\left(x_{P}\right) .
\end{aligned}
$$


$\left(y_{K}(P)\right)_{K \in \mathcal{V}_{P}}$ is a set of weights around $P$ which will be precised later. The reconstructed gradient is

$$
\mathbf{p}_{\mathbf{e}}=\left(\frac{u_{E}-u_{W}}{h_{e}}-\alpha_{e} \frac{u_{N}-u_{S}}{m(e)}\right) \mathbf{n}_{\mathbf{e}}+\frac{u_{N}-u_{S}}{m(e)} \mathbf{t}_{\mathbf{e}}
$$

where $h_{e}=W E . \mathbf{n}_{e}, m(e)=S N . \mathbf{t}_{e}$ and $\alpha_{e}=\tan \left(\mathbf{n}_{e}, W E\right)=\frac{W E . \mathbf{t}_{e}}{W E . \mathbf{n}_{e}}$.

If $e \in \Gamma_{h}$ and $\mathbf{n}_{e}=\mathbf{n}_{\text {ext }}$ (i.e. $E$ is reduced to the edge), then we take naturally

$$
u_{E}=g\left(x_{E}\right) .
$$

Remark that we took $u_{W}=g\left(x_{W}\right)$ if $\mathbf{n}_{e}=-\mathbf{n}_{\text {ext }}$.

Using this gradient in the flux function yields the numerical flux of diffusion.

$$
\begin{aligned}
\phi_{e}^{D}\left(u_{h}\right) & =\frac{1}{m(e)} \int_{e}\left(A \mathbf{p}_{e}\right) \cdot \mathbf{n}_{e} \mathrm{~d} s=\left(A_{e} \mathbf{p}_{e}\right) \cdot \mathbf{n}_{e} \\
& =\lambda_{e} \frac{u_{E}-u_{W}}{h_{e}}+\beta_{e} \frac{u_{N}-u_{S}}{m(e)},
\end{aligned}
$$

where $A_{e}=\frac{1}{m(e)} \int_{e} A \mathrm{~d} s=\left[\begin{array}{ll}\lambda_{e} & \mu_{e} \\ \mu_{e} & \nu_{e}\end{array}\right]$ in $\left(\mathbf{n}_{e}, \mathbf{t}_{e}\right)$, and $\beta_{e}=\mu_{e}-\alpha_{e} \lambda_{e}$.

\subsection{The discrete operator}

At last, the approximation $u_{h}$ is the solution of the following discrete problem

$$
\sum_{e \in T_{h}} s_{K e}\left(\phi_{e}^{C}\left(u_{h}\right)-\phi_{e}^{D}\left(u_{h}\right)\right) m(e)=m(K) f_{K} .
$$

Denoting by $\mathcal{P}_{0}\left(T_{h}\right)$ the space of the functions constant on each cell $K$, we can define a discrete operator $\mathcal{L}_{h}$ on $\mathcal{P}_{0}\left(T_{h}\right)$ and for a given boundary condition $g$ in $V(\Gamma)$ by

$$
\mathcal{L}_{h}\left(u_{h}, g\right)_{\mid K}=\frac{1}{m(K)} \sum_{e \in T_{h}} s_{K e}\left(\phi_{e}^{C}\left(u_{h}\right)-\phi_{e}^{D}\left(u_{h}\right)\right) m(e)
$$

such that $u_{h}$ is the solution in $\mathcal{P}_{0}\left(T_{h}\right)$ of $\mathcal{L}_{h}\left(u_{h}, g\right)=f_{h}$, where $f_{h}$ designates the function in $\mathcal{P}_{0}\left(T_{h}\right)$ of value $f_{K}$ on $K$.

\section{WEAK CONSISTENCY}

The consistency error as defined below is the difference between the exact flux calculated for a given function $u \in W^{2, p}(\Omega)$ on each edge of the mesh and the corresponding numerical flux evaluated with the $L^{2}$ projection $\pi_{h} u$ of $u$ on $\mathcal{P}_{0}\left(T_{h}\right)$. A similar definition has been introduced by Gallouët et al. in [9,10] or by Herbin in [12] but assuming the $C^{2}$ regularity of $u$ and using pointwise values of $u$ instead of mean values to define the discrete flux.

\subsection{Definition}

Definition 3.1. For $u \in W^{2, p}(\Omega)$, the consistency error $R_{h}(u)$ is the piecewise constant function of value $R_{e}$ on each of the diamond-shaped co-volumes $\chi_{e}$, such that

$$
R_{e}(u)=\left(\phi_{e}^{C}\left(\pi_{h} u\right)-\phi_{e}^{D}\left(\pi_{h} u\right)\right)-\left(\phi_{e}^{C}(u)-\phi_{e}^{D}(u)\right) .
$$


$\pi_{h} u$ is the $L^{2}$ projection of $u$ on $\mathcal{P}_{0}\left(T_{h}\right)$ and $\phi_{e}^{D / C}\left(\pi_{h} u\right)$ are the fluxes defined like in Section 2 , but for the discrete function $\pi_{h} u$ and the boundary condition $g=\gamma_{0}(u)$.

The scheme (10) [or the operator $\mathcal{L}_{h}(11)$ ] is said to be weakly consistent if

$$
\forall u \in W^{2, p}(\Omega), \quad\left\|R_{h}(u)\right\|_{L^{2}} \underset{h \rightarrow 0}{\longrightarrow} 0 .
$$

Remark. A strong consistency error between the discrete operator $\mathcal{L}_{h}$ and the continuous one $\mathcal{L}=-\operatorname{div}(A \nabla \cdot-$ v.) can be worked out as follows:

$$
\begin{aligned}
m(K)\left(\pi_{h}(\mathcal{L} u)_{\mid K}-\mathcal{L}_{h}\left(\pi_{h} u, \gamma_{0} u\right)_{\mid K}\right) & =\int_{K}\left(\mathcal{L} u-\mathcal{L}_{h}\left(\pi_{h} u, \gamma_{0} u\right)\right) \mathrm{d} x \\
& =\sum_{e \in \partial K} s_{K e} R_{e}(u) m(e)
\end{aligned}
$$

We find after a short calculation

$$
\left\|\pi_{h}(\mathcal{L} u)-\mathcal{L}_{h}\left(\pi_{h} u, \gamma_{0} u\right)\right\|_{L^{2}(\Omega)} \leq \frac{C}{h}\left\|R_{h}(u)\right\|_{L^{2}}
$$

But generally, the best estimate of $R_{h}$ should only be of order $h$. Hence, the finite volume scheme (10) may not be consistent in the usual sense.

\subsection{A weak consistency sufficient condition}

The weak consistency of the discrete operator (11) is characterized by some properties of the node interpolation weights $y_{K}(P)$ with the following lemma.

Theorem 3.1. If the three following conditions are fulfilled

$$
\begin{gathered}
\forall P \in N_{h}^{\star}, \quad \sum_{K \in \mathcal{V}_{P}} y_{K}(P)=1, \\
\forall P \in N_{h}^{\star}, \quad \sum_{K \in \mathcal{V}_{P}} y_{K}(P)\left(x_{K}-x_{P}\right)=0, \\
\forall h>0, \quad \forall P \in N_{h}^{\star}, \quad \max _{K \in \mathcal{V}_{P}}\left|y_{K}(P)\right|<C,
\end{gathered}
$$

then $\mathcal{L}_{h}$ is weakly consistent and the error verifies the following estimate:

$$
\left\|R_{h}(u)\right\|_{L^{2}} \leq C\|u\|_{W^{2, p}} h
$$

Remark. The two conditions $(12,13)$ characterize a projection which preserves the linear functions. The third condition (14) characterizes a uniformly (with respect to $h$ ) bounded projection. 
Proof of Theorem 3.1. We shall use the following lemma.

Lemma 3.1. For $u \in W^{1, p}(\mathcal{O})$ where $\mathcal{O}$ is a convex domain of $\mathbb{R}^{2}$ and $p>2$,

$$
\forall x, y \in \overline{\mathcal{O}}, \quad|u(x)-u(y)| \leq C\|\nabla u\|_{L^{p}(\mathcal{O})} \frac{\delta(\mathcal{O})}{m(\mathcal{O})^{1 / p}} .
$$

$\delta(\mathcal{O})$ and $m(\mathcal{O})$ are the diameter and the surface of $\mathcal{O} . C$ depends only on $p$.

Proof of Lemma 3.1. Remark that for $x \in \overline{\mathcal{O}}, u(x)$ makes sense because of the continuous imbedding of $W^{1, p}(\mathcal{O})$ into $C^{0}(\overline{\mathcal{O}})$. Of course $u$ denotes the continuous representative of $u \in W^{1, p}(\mathcal{O})$.

We begin by proving the result for the functions $u$ of $C^{\infty}(\overline{\mathcal{O}})$. For $x, x_{0} \in \overline{\mathcal{O}}$, we have

$$
\begin{aligned}
u\left(x_{0}\right)-u(x) & =\int_{0}^{1} \frac{\mathrm{d}}{\mathrm{d} t}\left(u\left(x+t\left(x_{0}-x\right)\right)\right) \mathrm{d} t \\
\left|u\left(x_{0}\right)-u(x)\right| & \leq \int_{0}^{1}\left|\nabla u\left(x+t\left(x_{0}-x\right)\right) \cdot\left(x_{0}-x\right)\right| \mathrm{d} t \\
& \leq \delta(\mathcal{O}) \int_{0}^{1} \sum_{i=1,2}\left|\partial_{i} u\left(x+t\left(x_{0}-x\right)\right)\right| \mathrm{d} t
\end{aligned}
$$

because $\left|x_{i 0}-x_{i}\right| \leq \delta(\mathcal{O})$ for $i=1,2$. Noting $\langle u\rangle_{\mathcal{O}}$ the average value of $u$ on $\mathcal{O}$, we get

$$
\begin{aligned}
\left|\langle u\rangle_{\mathcal{O}}-u(x)\right| & \leq \frac{\delta(\mathcal{O})}{m(\mathcal{O})} \int_{x_{0} \in \mathcal{O}} \int_{0}^{1} \sum_{i=1,2}\left|\partial_{i} u\left(x+t\left(x_{0}-x\right)\right)\right| \mathrm{d} t \mathrm{~d} x_{0} \\
& \leq \frac{\delta(\mathcal{O})}{m(\mathcal{O})} \int_{0}^{1} \sum_{i=1,2}\left(\int_{y \in x+t(\mathcal{O}-x)}\left|\partial_{i} u(y)\right| \frac{\mathrm{d} y}{t^{2}}\right) \mathrm{d} t
\end{aligned}
$$

since $t^{2} \mathrm{~d} x=\mathrm{d} y$ if we set $y=x+t\left(x_{0}-x\right)$. The Hölder's inequality gives

$$
\int_{y \in x+t(\mathcal{O}-x)}\left|\partial_{i} u(y)\right| \mathrm{d} y \leq\left(\int_{\mathcal{O}}\left|\partial_{i} u(y)\right|^{p} \mathrm{~d} y\right)^{1 / p}\left(t^{2} m(\mathcal{O})\right)^{1-1 / p},
$$

since $x+t(\mathcal{O}-x) \subset \mathcal{O}$ for $t \in] 0,1[$. Hence, it is deduced

$$
\begin{aligned}
\left|\langle u\rangle_{\mathcal{O}}-u(x)\right| & \leq \frac{\delta(\mathcal{O})}{m(\mathcal{O})}\|\nabla u\|_{L^{p}(\mathcal{O})} \int_{0}^{1} \frac{\left(t^{2} m(\mathcal{O})\right)^{1-1 / p}}{t^{2}} \mathrm{~d} t \\
& \leq \frac{p}{p-2} \frac{\delta(\mathcal{O})}{m(\mathcal{O})^{1 / p}}\|\nabla u\|_{L^{p}(\mathcal{O})}
\end{aligned}
$$

and then for $x, y \in \overline{\mathcal{O}}$, using the triangular inequality, we have

$$
|u(x)-u(y)| \leq C\|\nabla u\|_{L^{p}(\mathcal{O})} \frac{\delta(\mathcal{O})}{m(\mathcal{O})^{1 / p}},
$$

where $C$ depends only on $p$.

For $u \in W^{1, p}(\mathcal{O})$, we use a sequence $\left(u_{n}\right)$ in $C^{\infty}(\overline{\mathcal{O}})$ such that $u_{n} \longrightarrow u$ in $W^{1, p}(\mathcal{O})$ and in $C^{0}(\overline{\mathcal{O}})$. 
Following of the proof of Theorem 3.1. Afterwards, the error of consistency along an edge can be written as follows:

$$
\begin{aligned}
R_{e}(u) & =\left(\phi_{e}^{C}\left(\pi_{h} u\right)-\phi_{e}^{C}(u)\right)-\left(\phi_{e}^{D}\left(\pi_{h} u\right)-\phi_{e}^{D}(u)\right) \\
& =R_{e}^{C}(u)-R_{e}^{D}(u)
\end{aligned}
$$

where

$$
\begin{aligned}
R_{e}^{C}(u) & =\frac{1}{m(e)} \int_{e}\left(\mathbf{v} \cdot \mathbf{n}_{e}\right)\left(\bar{u}_{W}-u\right) \mathrm{d} s \\
R_{e}^{D}(u) & =\frac{1}{m(e)} \int_{e}\left(A \mathbf{p}_{e}\right) \cdot \mathbf{n}_{e}-(A \nabla u) \cdot \mathbf{n}_{e} d s .
\end{aligned}
$$

We note

$$
\begin{cases}\bar{u}_{K}=\pi_{h} u_{\mid K}=\langle u\rangle_{K} & \text { for all } K \in T_{h} \\ \bar{u}_{K}=u\left(x_{K}\right) & \text { for each midpoint } x_{K} \text { of a boundary edge, } \\ \bar{u}_{P}=\sum_{K \in \mathcal{V}_{P}} y_{K}(P) \bar{u}_{K} & \text { for all } P \in N_{h}^{\star} \\ \bar{u}_{P}=u\left(x_{P}\right) & \text { for each } P \in N_{h} \cap \Gamma\end{cases}
$$

\subsubsection{Error on the flux of convection}

For any $e \in S_{h}$, if $W \in T_{h}$, then for any $s \in e \subset \bar{W}$ and $x \in W$, by application of Lemma 3.1 to the function $u$ of $W^{1, p}$ on the convex $W$, and using hypotheses $(2)\left(\delta(W) \leq h\right.$ and $\left.m(W) \geq \alpha h^{2}\right)$,

$$
\begin{aligned}
|u(x)-u(s)| & \leq C\|\nabla u\|_{L^{p}(W)} \frac{\delta(W)}{m(W)^{1 / p}} \leq C\|\nabla u\|_{L^{p}(W)} h^{1-2 / p} \\
\left|\bar{u}_{W}-u(s)\right| & \leq \frac{1}{m(W)} \int_{W}|u(x)-u(s)| \mathrm{d} x \leq C\|\nabla u\|_{L^{p}(W)} h^{1-2 / p}
\end{aligned}
$$

Otherwise, $\bar{u}_{W}=u\left(x_{W}\right)$, and then for any $s \in e$ we also have by application of Lemma 3.1 on $E$, because $x_{W}, s \in \bar{E}$,

$$
\left|\bar{u}_{W}-u(s)\right| \leq C\|\nabla u\|_{L^{p}(E)} h^{1-2 / p} .
$$

At last we have

$$
\left|R_{e}^{C}(u)\right| \leq\|\mathbf{v}\|_{L^{\infty}(\Omega)} \frac{1}{m(e)} \int_{e}\left|u(s)-\bar{u}_{W}\right| \mathrm{d} s \leq C\|\nabla u\|_{L^{p}(W \cup E)} h^{1-2 / p} .
$$

As a consequence, using the hypotheses $(2)\left(m\left(\chi_{e}\right)=\frac{m(e) h_{e}}{2} \leq h^{2}\right.$ and Card $\left.\partial K \leq \gamma\right)$, we have

$$
\begin{aligned}
\left\|R_{h}^{C}(u)\right\|_{L^{p}(\Omega)}^{p}=\sum_{e \in S_{h}}\left|R_{e}^{C}(u)\right|^{p} m\left(\chi_{e}\right) & \leq C \sum_{e \in S_{h}}\|\nabla u\|_{L^{p}(W \cup E)}^{p} h^{p-2} h^{2} \\
& \leq C h^{p} \sum_{K \in T_{h}}\|\nabla u\|_{L^{p}(K)}^{p} \operatorname{Card} \partial K \\
& \leq C\|\nabla u\|_{L^{p}(\Omega)}^{p} h^{p},
\end{aligned}
$$

and then

$$
\left\|R_{h}^{C}(u)\right\|_{L^{2}(\Omega)} \leq m(\Omega)^{1-2 / p}\left\|R_{h}(u)\right\|_{L^{p}(\Omega)} \leq C\|\nabla u\|_{L^{p}(\Omega)} h .
$$


3.2.2. Error on the flux of diffusion

On any edge $e$ in $S_{h}$, we have

$$
\left(A(s) \mathbf{p}_{e}\right) \cdot \mathbf{n}_{e}=\lambda(s) \frac{\bar{u}_{E}-\bar{u}_{W}}{h_{e}}+\beta(s) \frac{\bar{u}_{N}-\bar{u}_{S}}{m(e)} .
$$

The exact flux can also be divided similarly:

$$
(A(s) \nabla u(s)) \cdot \mathbf{n}_{e}=\lambda(s) \nabla u(s) \cdot\left(\mathbf{n}_{e}+\alpha_{e} \mathbf{t}_{e}\right)+\beta(s) \nabla u(s) \cdot \mathbf{t}_{e} .
$$

We note that $h_{e}\left(\mathbf{n}_{e}+\alpha_{e} \mathbf{t}_{e}\right)=x_{E}-x_{W}$ and $m(e) \mathbf{t}_{e}=x_{N}-x_{S}$, and so

$$
\begin{aligned}
\left|R_{e}^{D}(u)\right|= & \frac{1}{m(e)} \int_{e}\left|\lambda(s)\left(\frac{\nabla u(s) \cdot\left(x_{E}-x_{W}\right)-\left(\bar{u}_{E}-\bar{u}_{W}\right)}{h_{e}}\right)\right| \mathrm{d} s \\
& +\frac{1}{m(e)} \int_{e}\left|\beta(s)\left(\frac{\nabla u(s) \cdot\left(x_{N}-x_{S}\right)-\left(\bar{u}_{N}-\bar{u}_{S}\right)}{m(e)}\right)\right| \mathrm{d} s .
\end{aligned}
$$

We shall use the following lemma.

Lemma 3.2. (corollary of Lemma 3.1) For $u \in W^{2, p}(\mathcal{O})$ where $\mathcal{O}$ is a convex domain of $\mathbb{R}^{2}$ and $p>2$, for all $x, y \in \overline{\mathcal{O}}$ let be

$$
\begin{aligned}
\mathcal{I}(x, y) & =u(x)-u(y)-\nabla u(y) \cdot(x-y) \\
& =\int_{0}^{1}(\nabla u(y+\sigma(x-y))-\nabla u(y)) \cdot(x-y) \mathrm{d} \sigma .
\end{aligned}
$$

We have

$$
|\mathcal{I}(x, y)| \leq C \frac{\delta(\mathcal{O})^{2}}{m(\mathcal{O})^{1 / p}}\left\|\nabla^{2} u\right\|_{L^{p}(\mathcal{O})}
$$

( $C$ depends only on $p$ ).

Proof of Lemma 3.2. Like in Lemma 3.1, $\partial_{i} u \in C^{0}(\overline{\mathcal{O}})$ for $u \in W^{2, p}(\mathcal{O})$. The result is obvious by applying Lemma 3.1 to the functions $\partial_{i} u(i=1,2)$.

End of the proof of Theorem 3.1. $\langle.\rangle_{X}$ still denotes the average value on $X$. Consider the following notations for any $s \in \Omega$ :

$$
\begin{array}{ll}
\text { if } K \in T_{h}, & \mathcal{I}_{K}(s)=\bar{u}_{K}-u(s)-\nabla u(s) .\left(x_{K}-s\right)=\langle\mathcal{I}(., s)\rangle_{K}, \\
\text { if } x_{K} \in \Gamma, & \mathcal{I}_{K}(s)=\bar{u}_{K}-u(s)-\nabla u(s) .\left(x_{K}-s\right)=\mathcal{I}\left(x_{K}, s\right), \\
\text { if } P \in \Gamma, & \mathcal{I}_{P}(s)=\bar{u}_{P}-u(s)-\nabla u(s) \cdot\left(x_{P}-s\right)=\mathcal{I}\left(x_{P}, s\right), \\
\text { if } P \in N_{h}^{\star}, & \mathcal{I}_{P}(s)=\bar{u}_{P}-u(s)-\nabla u(s) .\left(x_{P}-s\right)=\sum_{K \in \mathcal{V}_{P}} y_{K}(P)\langle\mathcal{I}(., s)\rangle_{K},
\end{array}
$$

where $\bar{u}_{K}$ and $\bar{u}_{P}$ are defined by (16). The last equality results of hypotheses $(12,13)$ of Theorem 3.1 , and then we can write for $e \in S_{h}$ and $s \in e$ :

$$
\begin{aligned}
\left|\nabla u(s) \cdot\left(x_{E}-x_{W}\right)-\left(\bar{u}_{E}-\bar{u}_{W}\right)\right| & =\left|\mathcal{I}_{E}(s)-\mathcal{I}_{W}(s)\right|, \\
\left|\nabla u(s) \cdot\left(x_{N}-x_{S}\right)-\left(\bar{u}_{N}-\bar{u}_{S}\right)\right| & =\left|\mathcal{I}_{N}(s)-\mathcal{I}_{S}(s)\right| .
\end{aligned}
$$


Hence, the local error on a given edge $e$ is

$$
\left|R_{e}^{D}(u)\right| \leq \frac{\sup _{e}|\lambda|}{m(e)} \int_{e} \frac{\left|\mathcal{I}_{E}(s)\right|+\left|\mathcal{I}_{W}(s)\right|}{h_{e}}+\frac{\sup _{e}|\beta|}{m(e)} \int_{e} \frac{\left|\mathcal{I}_{N}(s)\right|+\left|\mathcal{I}_{S}(s)\right|}{m(e)},
$$

and it remains to estimate separately the different errors $\mathcal{I}_{K}$ and $\mathcal{I}_{P}$ on a given edge $e \in S_{h}$.

The first case is $K=E$ or $W$. We have with Lemma 3.2 used as in the case of the flux of convection (using again $\delta(K) \leq h$ and $\left.m(K) \geq \alpha h^{2}\right)$

$$
\forall s \in e, \quad\left|\mathcal{I}_{K}(s)\right| \leq C\left\|\nabla^{2} u\right\|_{L^{p}(E \cup W)} h^{2-2 / p} .
$$

The second case is $P=N$ or $S \in \Gamma \cap N_{h}$, for which $s, x_{P} \in \bar{\chi}_{e}$. Consequently, Lemma 3.2 gives

$$
\forall s \in e, \quad\left|\mathcal{I}_{P}(s)\right|=\left|\mathcal{I}\left(x_{P}, s\right)\right| \leq C\left\|\nabla^{2} u\right\|_{L^{p}\left(\chi_{e}\right)} \frac{\delta\left(\chi_{e}\right)^{2}}{m\left(\chi_{e}\right)^{1 / p}} .
$$

The last case is $P=N$ or $S \in N_{h}^{\star}$. In that case, $\mathcal{I}_{P}(s)$ depends on all the $\mathcal{I}_{K}(s)$ for $K \in \mathcal{V}_{P}$. But since $\cup_{\mathcal{V}_{P}} K$ is not necessarily convex, we shall use the following decomposition:

$$
\mathcal{I}(x, y)=\mathcal{I}(x, z)+\mathcal{I}(z, y)+(\nabla u(z)-\nabla u(y)) \cdot(x-z)
$$

As a result, for $K \in \mathcal{V}_{P}$, we get for all $s \in e$,

$$
\mathcal{I}_{K}(s)=\langle\mathcal{I}(., s)\rangle_{K}=\left\langle\mathcal{I}\left(., x_{P}\right)\right\rangle_{K}+\mathcal{I}\left(x_{P}, s\right)+\left(\nabla u\left(x_{P}\right)-\nabla u(s)\right) \cdot\left\langle\left(.-x_{P}\right)\right\rangle_{K}
$$

Applying Lemma 3.2 on $K$ and $\chi_{e}$ for the two first terms, $\left\langle.-x_{P}\right\rangle_{K} \leq \delta(K)$ and Lemma 3.1 for $\partial_{i} u$ on $\chi_{e}$ for the last term, we have

$$
\begin{aligned}
\left|\mathcal{I}_{K}(s)\right| \leq & C\left\|\nabla^{2} u\right\|_{L^{p}(K)} \frac{\delta(K)^{2}}{m(K)^{1 / p}}+C\left\|\nabla^{2} u\right\|_{L^{p}\left(\chi_{e}\right)} \frac{\delta\left(\chi_{e}\right)^{2}}{m\left(\chi_{e}\right)^{1 / p}} \\
& +C\left\|\nabla^{2} u\right\|_{L^{p}\left(\chi_{e}\right)} \frac{\delta\left(\chi_{e}\right)}{m\left(\chi_{e}\right)^{1 / p}} \delta(K) .
\end{aligned}
$$

Hypothesis (14) of Theorem 3.1 and the geometric assumptions (2) yields finally

$$
\left|\mathcal{I}_{P}(s)\right| \leq C\left\|\nabla^{2} u\right\|_{L^{p}\left(\mathcal{V}_{P}\right)} h^{2-2 / p}+C\left\|\nabla^{2} u\right\|_{L^{p}\left(\chi_{e}\right)} h^{2-2 / p}
$$

At last, gathering the different contributions to calculate the error (17), we have

$$
\left|R_{e}^{D}(u)\right| \leq C\|A\|_{L^{\infty}(\Omega)}\left\|\nabla^{2} u\right\|_{L^{p}\left(\mathcal{V}_{N} \cup \mathcal{V}_{S}\right)} h^{1-2 / p}
$$

Completing the calculation as in the case of the flux of convection yields

$$
\begin{aligned}
\left\|R_{h}^{D}(u)\right\|_{L^{p}(\Omega)}^{p} & \leq C \sum_{e \in S_{h}}\left\|\nabla^{2} u\right\|_{L^{p}\left(\mathcal{V}_{N} \cup \mathcal{V}_{S}\right)}^{p} h^{p} \\
& \leq C \sum_{K \in T_{h}}\left\|\nabla^{2} u\right\|_{L^{p}(K)}^{p}\left(\operatorname{Card} \mathcal{V}_{N} \cup \mathcal{V}_{S}\right) h^{p} \leq C\left\|\nabla^{2} u\right\|_{L^{p}(\Omega)}^{p} h^{p}, \\
\left\|R_{h}^{D}(u)\right\|_{L^{2}(\Omega)} & \leq m(\Omega)^{1-2 / p}\left\|R_{h}^{D}(u)\right\|_{L^{p}(\Omega)} \leq C\left\|\nabla^{2} u\right\|_{L^{p}(\Omega)} h .
\end{aligned}
$$


At last, for any $u$ in $W^{2, p}(\Omega)$ we have proved:

$$
\left\|R_{h}(u)\right\|_{L^{2}(\Omega)} \leq C\|u\|_{W^{2, p}(\Omega)} h .
$$

\section{Remark.}

- This method yields the same result if the consistency is defined with a pointwise valued projection $\left(\pi_{h} u_{\mid K}=\right.$ $u\left(x_{K}\right)$ instead of $\left.\pi_{h} u_{\mid K}=\langle u\rangle_{K}\right)$.

- The inequalities resulting from the hypotheses on the mesh (2) only use $\alpha h^{2} \leq m(K)$ and Card $\partial K \leq \gamma$, except $(18,19)$. In this case, several terms have to be divided by $h_{e}$ or $m(e)$. Hence, the hypothesis $m(e) \geq \beta h$ is also necessary.

\subsection{A least squares interpolation to calculate the weights}

In this paragraph the couple $(x, y) \in \mathbb{R}^{2}$ will denote a couple of coordinates.

Let $w$ be an affine function defined on the reunion of the $K$ in $\mathcal{V}_{P}$ for $P$ in $N_{h}^{\star}$. In a system of coordinates of origin $P$ the function $w$ takes the following form.

$$
w(x, y)=a+b x+c y
$$

The least squares interpolation consists in choosing $a, b, c$ in order to minimize the quadratic function

$$
L_{P}(w)=\sum_{K \in \mathcal{V}_{P}}\left(u_{K}-w\left(x_{K}, y_{K}\right)\right)^{2} .
$$

Hence, $a, b, c$ are given by

$$
\sum_{K \in \mathcal{V}_{P}}\left(w\left(x_{K}, y_{K}\right)-u_{K}\right) \nabla_{a, b, c} w\left(x_{K}, y_{K}\right)=0 .
$$

Using $\nabla_{a, b, c} w\left(x_{K}, y_{K}\right)=\left[\begin{array}{c}1 \\ x_{K} \\ y_{K}\end{array}\right]$, it yields

$$
\left[\begin{array}{ccc}
n_{P} & R_{x} & R_{y} \\
R_{x} & I_{x x} & I_{x y} \\
R_{y} & I_{x y} & I_{y y}
\end{array}\right]\left[\begin{array}{l}
a \\
b \\
c
\end{array}\right]=\left[\begin{array}{llll}
\sum u_{K} & \sum x_{K} u_{K} & \sum y_{K} u_{K}
\end{array}\right]^{T},
$$

with

$$
R_{x}=\sum x_{K}, \quad R_{y}=\sum y_{K}, \quad I_{x x}=\sum x_{K}^{2}, \quad I_{x y}=\sum x_{K} y_{K}, \quad I_{y y}=\sum y_{K}^{2} .
$$

$n_{P}=$ Card $\mathcal{V}_{P}$ is the number of cell around $P$. All the sums are performed over $\mathcal{V}_{P}$. Afterwards, noting that $w(P)=a$, the least squares approximation yields the following node value:

$$
a=u_{P}=\sum_{K \in \mathcal{V}_{P}} y_{K}(P) u_{K}, \text { with } y_{K}(P)=\frac{1+\lambda_{x} x_{K}+\lambda_{y} y_{K}}{n+\lambda_{x} R_{x}+\lambda_{y} R_{y}}
$$

and where

$$
D=I_{x x} I_{y y}-I_{x y}^{2}, \quad \lambda_{x}=\frac{I_{x y} R_{y}-I_{y y} R_{x}}{D}, \quad \lambda_{y}=\frac{I_{x y} R_{x}-I_{x x} R_{y}}{D} .
$$

Remark that $D$ cannot be equal to zero because $I_{x x} I_{y y}>I_{x y}^{2}$ (Schwarz' inequality for the two vectors $X$ and $Y$ of coordinates of the centres of the $K$ of $\mathcal{V}_{P}$, which are linearly independent because the centres $\left(x_{K}, y_{K}\right)$ cannot be all aligned with $P$ ).

The first two hypotheses $(12,13)$ of 3.1 are obviously satisfied. To ensure the weak consistency, it remains the third condition (14) to be verified. 
Setting $R_{x}=n_{P} x_{G}$ and $R_{y}=n_{P} y_{G}$, we have

$$
\begin{aligned}
& I_{x x}=n_{P}\left(\sigma_{x}^{2}+x_{G}^{2}\right), \quad \text { with } \quad \sigma_{x}^{2}=\frac{1}{n_{P}} \sum_{K \in \mathcal{V}_{P}}\left(x_{K}-x_{G}\right)^{2}, \\
& I_{y y}=n_{P}\left(\sigma_{y}^{2}+y_{G}^{2}\right), \quad \text { with } \quad \sigma_{y}^{2}=\frac{1}{n_{P}} \sum_{K \in \mathcal{V}_{P}}\left(y_{K}-y_{G}\right)^{2}, \\
& I_{x y}=n_{P}\left(\sigma_{x y}^{2}+x_{G} y_{G}\right), \quad \text { with } \quad \sigma_{x y}^{2}=\frac{1}{n_{P}} \sum_{K \in \mathcal{V}_{P}}\left(x_{K}-x_{G}\right)\left(y_{K}-y_{G}\right) .
\end{aligned}
$$

With these notations, a calculation yields

$$
y_{K}(P)=\frac{1}{n_{P}}\left(1+\frac{x_{G}\left(x_{G}-x_{K}\right)}{\sigma_{x}^{2}}+\frac{y_{G}\left(y_{G}-y_{K}\right)}{\sigma_{y}^{2}}\right) .
$$

Hence, hypothesis (14) is a direct consequence of the regularity assumptions (2) on the mesh: $\left|x_{G}\right| \leq \max \delta(K)$, $\left|x_{G}-x_{K}\right| \leq\left|x_{G}-x_{P}\right|+\left|x_{P}-x_{K}\right| \leq 2 \max \delta(K)$ and $\sum_{K \in \mathcal{V}_{P}}\left(x_{G}-x_{K}\right)^{2} \geq C h^{2}$ (see [6] for the details).

Let us finally mention that this set of weights has been successfully used for various numerical experiments by different authors $[2,14,22]$.

\section{Coercivity of the Discrete operator}

\subsection{Definition}

Definition 4.1. The discrete operator $\mathcal{L}_{h}$ is said to be coercive if there exists $\alpha>0$ such that

$$
\forall h>0, \forall \varepsilon_{h} \in \mathcal{P}_{0}\left(T_{h}\right), \quad\left(\mathcal{L}_{h}\left(\varepsilon_{h}, 0\right), \varepsilon_{h}\right)_{L^{2}} \geq 2 \alpha\left\|\varepsilon_{h}\right\|_{1,0}^{2},
$$

where

$$
\left\|\varepsilon_{h}\right\|_{1,0}=\left(\sum_{e \in S_{h}}\left(\frac{\varepsilon_{E}-\varepsilon_{W}}{h_{e}}\right)^{2} m\left(\chi_{e}\right)\right)^{1 / 2}
$$

We assume the following convention for $e \in S_{h}$ :

$$
\varepsilon_{E}=0 \text { if } \mathbf{n}_{e}=\mathbf{n}_{\text {ext }} \text { and } \varepsilon_{W}=0 \text { if } \mathbf{n}_{e}=-\mathbf{n}_{\text {ext }} .
$$

We recall that $h_{e}=\left(x_{E}-x_{W}\right) \cdot \mathbf{n}_{e}$ and remark that (22) actually defines a norm on $\mathcal{P}_{0}\left(T_{h}\right)$ (see for instance Lemma 5.1).

Note that a straightforward consequence of the coercivity of $\mathcal{L}_{h}$ is the well-posedness of the discrete problem $\mathcal{L}_{h}\left(u_{h}, g\right)=f_{h}$.

\subsection{A sufficient coercivity condition}

Throughout this part, we would note $\mathcal{L}_{h} u_{h}$ for $\mathcal{L}_{h}\left(u_{h}, 0\right)$.

The main issue of this part is to provide a condition on the tangential part of the gradient to ensure the coercivity of the discrete operator. 
$Q\left(u_{h}\right)=\left(\mathcal{L}_{h} u_{h}, u_{h}\right)_{L^{2}}$ can be obviously split into a part of diffusion $\mathcal{Q}^{D}\left(u_{h}\right)$ and a part of convection $\mathcal{Q}^{C}\left(u_{h}\right)$.

$$
\begin{aligned}
\mathcal{Q}\left(u_{h}\right)=\left(\mathcal{L}_{h} u_{h}, u_{h}\right)_{L^{2}} & =\sum_{K \in T_{h}} u_{K} \sum_{e \in \partial K} s_{K e}\left(\phi_{e}^{C}\left(u_{h}\right)-\phi_{e}^{D}\left(u_{h}\right)\right) m(e) \\
& =\sum_{e \in S_{h}}\left(\phi_{e}^{D}\left(u_{h}\right)-\phi_{e}^{C}\left(u_{h}\right)\right) \frac{u_{E}-u_{W}}{h_{e}} 2 m\left(\chi_{e}\right) \\
& =\mathcal{Q}^{D}\left(u_{h}\right)+\mathcal{Q}^{C}\left(u_{h}\right) .
\end{aligned}
$$

Remark that $m\left(\chi_{e}\right)=\frac{1}{2} m(e) h_{e}$.

4.2.1. Coercivity estimate for the flux of convection

Recalling the expression (5) of $\phi^{C}\left(u_{h}\right)$, we have

$$
\begin{aligned}
\mathcal{Q}^{C}\left(u_{h}\right) & =-\sum_{e \in S_{h}} u_{W} \mathbf{v}_{e} \cdot \mathbf{n}_{e}\left(u_{E}-u_{W}\right) m(e) \\
& =\frac{1}{2} \sum_{e \in S_{h}} \mathbf{v}_{e} \cdot \mathbf{n}_{e}\left(\left(u_{E}-u_{W}\right)^{2}+\left(u_{W}^{2}-u_{E}^{2}\right)\right) m(e)
\end{aligned}
$$

The first term is obviously positive and the second one is positive thanks to the hypothesis $\operatorname{div} \mathbf{v} \geq 0$ :

$$
\begin{aligned}
\sum_{e \in S_{h}} \mathbf{v}_{e} \cdot \mathbf{n}_{e}\left(u_{W}^{2}-u_{E}^{2}\right) m(e) & =\sum_{K \in T_{h}} u_{K}^{2} \sum_{e \in \partial K} \mathbf{v}_{e} \cdot \mathbf{n}_{K e} m(e) \\
& =\sum_{K \in T_{h}} u_{K}^{2} \int_{K} \operatorname{div} \mathbf{v} \geq 0
\end{aligned}
$$

Hence, we have proved

$$
\mathcal{Q}^{C} \geq 0 \text { and so } \mathcal{Q} \geq \mathcal{Q}^{D}
$$

\subsubsection{Coercivity estimate for the flux of diffusion}

Denoting by $\mathbf{p}_{h}^{\perp}$ the component of the discrete gradient (9) which appears in the definition (22) of $\|\cdot\|_{1,0}$, $\mathcal{Q}^{D}\left(u_{h}\right)$ can be written as

$$
\mathcal{Q}^{D}\left(u_{h}\right)=\sum_{e \in S_{h}}\left(A_{e} \mathbf{p}_{e}^{\perp}\right) \cdot \mathbf{p}_{e} 2 m\left(\chi_{e}\right)=2\left(A_{h} \mathbf{p}_{h}^{\perp}, \mathbf{p}_{h}\right)_{L^{2}}
$$

where

- $\mathbf{p}_{e}^{\perp}=\frac{u_{E}-u_{W}}{h_{e}} \mathbf{n}_{e}$ and $\mathbf{p}_{e}$ is given by (9) on $\chi_{e}$,

- $A_{h}$ is the piecewise constant function of value $A_{e}$ on $\chi_{e}$ for each $e \in S_{h}$.

In the continuous case, the operator is easily proved to be coercive since the quadratic form $(A \nabla u, \nabla u)_{L^{2}}$ is a norm of $\nabla u$, equivalent to the $L_{2}$ norm of $\nabla u$. This last result comes out as a natural consequence of the strict positivity of the matrix $A$. Similarly in the discrete case, the bilinear form $\left(A_{h} \mathbf{p}_{h}^{\perp}, \mathbf{p}_{h}\right)_{L^{2}}$ being a norm for $\mathbf{p}_{h}^{\perp}$, equivalent to the $L_{2}$ norm of $\mathbf{p}_{h}^{\perp}$, ensure the coercivity of the discrete operator as defined by (4.1). So $\left(A_{h} \mathbf{p}_{h}^{\perp}, \mathbf{p}_{h}\right)_{L^{2}}$ is split to be minorated into the norm $\left(A_{h} \mathbf{p}_{h}^{\perp}, \mathbf{p}_{h}^{\perp}\right)_{L^{2}}$ and a remainder which is to be controlled:

$$
\left(A_{h} \mathbf{p}_{h}^{\perp}, \mathbf{p}_{h}\right)_{L^{2}} \geq\left(A_{h} \mathbf{p}_{h}^{\perp}, \mathbf{p}_{h}^{\perp}\right)_{L^{2}}-\left|\left(A_{h} \mathbf{p}_{h}^{\perp}, \mathbf{p}_{h}-\mathbf{p}_{h}^{\perp}\right)_{L^{2}}\right|,
$$

and it can be completed the following lemma which will be used in Section 6 . 
Lemma 4.1. If there exists a positive constant $\gamma<1$ such that $\forall u_{h} \in \mathcal{P}_{0}\left(T_{h}\right)$,

$$
\sum_{e \in S_{h}^{\star}} \frac{\beta_{e}^{2}}{\lambda_{e}^{2}}\left(\frac{u_{N}-u_{S}}{m(e)}\right)^{2} \lambda_{e} m\left(\chi_{e}\right) \leq \gamma \sum_{e \in S_{h}}\left(\frac{u_{E}-u_{W}}{h_{e}}\right)^{2} \lambda_{e} m\left(\chi_{e}\right)
$$

then the discrete finite volume operator (11) is coercive:

$$
\forall u_{h} \in \mathcal{P}_{0}\left(T_{h}\right), \quad \mathcal{Q}^{D}\left(u_{h}\right) \geq 2 \lambda_{\min } \frac{1-\gamma}{2}\left\|u_{h}\right\|_{1,0}^{2}
$$

- $\lambda_{\min }=\inf _{e \in S_{h}} \lambda_{e} \geq C>0$.

- We recall that $A_{e}=\left[\begin{array}{ll}\lambda_{e} & \mu_{e} \\ \mu_{e} & \nu_{e}\end{array}\right]$ in the basis $\left(\mathbf{n}_{e}, \mathbf{t}_{e}\right)$, and $\beta_{e}=\mu_{e}-\alpha_{e} \lambda_{e}\left(\alpha_{e}=\tan \left(\mathbf{n}_{e},\left(x_{E}-x_{W}\right)\right)\right.$.

Proof. $\mathcal{Q}^{D}\left(u_{h}\right)$ has been shown in $(24)$ to be eventually split into a norm of $\mathbf{p}_{h}^{\perp}$ and a remainder. Afterwards, we have (remark that $u_{N}-u_{S}=0$ for $e \in \Gamma_{h}$ )

$$
\begin{aligned}
\left(A_{h} \mathbf{p}_{h}^{\perp}, \mathbf{p}_{h}^{\perp}\right)_{L^{2}} & =\sum_{e \in S_{h}} \lambda_{e}\left(\frac{u_{E}-u_{W}}{h_{e}}\right)^{2} m\left(\chi_{e}\right) \\
\left|\left(A_{h} \mathbf{p}_{h}^{\perp}, \mathbf{p}_{h}-\mathbf{p}_{h}^{\perp}\right)_{L^{2}}\right|= & \left|\sum_{e \in S_{h}^{\star}} \beta_{e} \frac{u_{E}-u_{W}}{h_{e}} \frac{u_{N}-u_{S}}{m(e)} m\left(\chi_{e}\right)\right| \\
\leq & \sum_{e \in S_{h}^{\star}} \frac{1}{2}\left(\left(\frac{u_{E}-u_{W}}{h_{e}}\right)^{2}\right. \\
& \left.+\left(\frac{\beta_{e}}{\lambda_{e}}\right)^{2}\left(\frac{u_{N}-u_{S}}{m(e)}\right)^{2}\right)_{e} m\left(\chi_{e}\right) \\
\leq & \frac{1+\gamma}{2} \sum_{e \in S_{h}^{\star}} \lambda_{e}\left(\frac{u_{E}-u_{W}}{h_{e}}\right)^{2} m\left(\chi_{e}\right)
\end{aligned}
$$

[using hypothesis (25)]. Hence, the conclusion is being completed as a natural consequence of (24) and of the hypothesis $\gamma<1$ :

$$
\frac{1}{2} \mathcal{Q}^{D}\left(u_{h}\right) \geq \frac{1-\gamma}{2}\left(A \mathbf{p}_{h}^{\perp}, \mathbf{p}_{h}^{\perp}\right)_{L^{2}} \geq \lambda_{\min } \frac{1-\gamma}{2}\left\|u_{h}\right\|_{1,0}^{2}
$$

Remark. The hypothesis of Lemma 4.1 expresses the fact that the contribution of the tangential gradient $\left(u_{N}-u_{S}\right)$ should be controlled by the normal gradient.

- It becomes obvious on regular meshes, such that there is no contributions of the tangential gradient: rectangles, triangles (VF4 scheme, see [12]), or more generally Voronoï meshes, for $A=\nu \mathrm{Id}$.

- It is quite easy to obtain on regular meshes of parallelograms, and consequently on smoothly deformed parallelograms (see Sect. 6).

- In case of a scalar diffusion, the following rough estimate can be derived from Lemma 4.1.

$$
\left|\frac{\alpha_{e} h_{e}}{m(e)}\right| \leq \frac{1}{N_{e}}
$$


where $N_{e}$ denotes the total number of cells which share $\bar{e}$. This very restrictive condition was shown, however, not to be necessary, by some numerical experiments, carried out on very unstructured meshes. They show a convergence rate of order $h$ in case of refined heterogeneous meshes of quadrangles an triangles (see $[22])$.

- Another approach of the coercivity is currently studied, which leads to an explicit geometrical condition, rather less restrictive than (26). But, verifying this condition needs a few additional geometric properties of the mesh. It is being tested on locally refined Cartesian meshes.

\section{ERROR ESTIMATE FOR THE DIAMOND CELL METHOD ON GENERAL MESHES}

\subsection{A discrete Poincaré's inequality}

To prove our main result, we shall need the following

Lemma 5.1. $\exists C>0$ such that $\forall h>0, \forall \varepsilon_{h} \in \mathcal{P}_{0}\left(T_{h}\right)$,

$$
\left\|\varepsilon_{h}\right\|_{1,0} \geq C\left\|\varepsilon_{h}\right\|_{L^{2}} .
$$

Proof. The demonstration is carried out with an analysis similar to the one of the continuous case (see [12]). A peculiar oriented direction $\mathcal{D}$ is previously sen. Thus any cell centre $K$ of $T_{h}$ is connected to an upstream (with respect to $\mathcal{D}$ ) centre $K_{0}$ of an edge of the boundary $\Gamma_{h}$ by a straight line of direction $\mathcal{D}$. This connection crosses a certain number $\mathcal{D}(K)$ of cells and their interfaces $e$. As a result, $u_{K}$ may be written as

$$
u_{K}=u_{K}-u_{K_{0}}=\sum_{e \in \mathcal{D}(K)}\left(u_{e^{+}}-u_{e^{-}}\right),
$$

where $e^{+}$and $e^{-}$denote the downstream and upstream cells along the edge $e$, with respect to the previous orientation and $u_{K_{0}}=0$. Afterwards, a Cauchy-Schwarz inequality procures the majoration of the square of this equality:

$$
\begin{aligned}
u_{K}^{2} & \leq\left(\sum_{e \in \mathcal{D}(K)} \frac{u_{e^{+}}-u_{e^{-}}}{h_{e}} \frac{h_{e}}{m\left(\chi_{e}\right)} m\left(\chi_{e}\right)\right)^{2} \\
& \leq \sum_{e \in \mathcal{D}(K)}\left(\frac{u_{e^{+}}-u_{e^{-}}}{h_{e}}\right)^{2} m\left(\chi_{e}\right) \sum_{e \in \mathcal{D}(K)}\left(\frac{h_{e}}{m\left(\chi_{e}\right)}\right)^{2} m\left(\chi_{e}\right) .
\end{aligned}
$$

The following estimate of the number of cells of $\mathcal{D}(K)$ has been proved in [12], remarking that hypotheses $(2)$ provide $\beta h \leq m(e) \leq h$ and $\alpha h^{2} \leq m(K) \leq C h^{2}$.

$$
\operatorname{Card} \mathcal{D}(K) \leq \frac{C}{h} \text { and } \frac{h_{e}^{2}}{m\left(\chi_{e}\right)}=\frac{2 h_{e}}{m(e)} \leq C .
$$

The Poincaré's inequality is then deduced from multiplying (28) by $m(K)$, summing over the cells $K$ and then swapping the two sums:

$$
\begin{aligned}
\sum_{K \in T_{h}} u_{K}^{2} m(K) & \leq \frac{C}{h} \sum_{K \in T_{h}} \sum_{e \in \mathcal{D}(K)} m(K)\left(\frac{u_{E}-u_{W}}{h_{e}}\right)^{2} m\left(\chi_{e}\right) \\
& \leq \frac{C}{h} \sum_{e \in S_{h}}\left(\sum_{K \in \mathcal{S}(e)} m(K)\right)\left(\frac{u_{E}-u_{W}}{h_{e}}\right)^{2} m\left(\chi_{e}\right) .
\end{aligned}
$$


The cells $K$ of $\mathcal{S}(e)$ are such that $e$ is in the upstream "shadow" of the cell $K(e \in \mathcal{D}(K))$. Consequently, $\mathcal{S}(e)$ collects the cells $K$ which cross the downstream connecting line from the edge $e$ to the boundary, with respect to $\mathcal{D}$ :

$$
e \in S_{h} \text { and } K \in \mathcal{S}(e) \Leftrightarrow K \in T_{h} \text { and } e \in \mathcal{D}(K)
$$

At last (see [12]),

$$
\operatorname{Card} \mathcal{S}(e) \leq \frac{C}{h} \text { and } m(K) \leq C h^{2}
$$

and the resulting inequality follows:

$$
\left\|u_{h}\right\|_{L^{2}}^{2} \leq C\left\|u_{h}\right\|_{1,0}^{2} .
$$

\subsection{Main result}

Theorem 5.1. If the conditions of consistency (12, 13, 14) and Lemma 4.1 of coercivity are fulfilled, then the diamond cell finite volume scheme (10) converges to the exact solution u of (1) with the following error estimate:

$$
\exists C>0, \forall h>0, \quad\left\|u_{h}-\pi_{h} u\right\|_{1,0}+\left\|u_{h}-u\right\|_{L^{2}}<C\|u\|_{W^{2, p}} h,
$$

where $\pi_{h} u$ denotes the $L^{2}$ projection of $u$ on $\mathcal{P}_{0}\left(T_{h}\right)$.

Proof of Theorem 5.1. Let $u$ be the continuous solution, $u_{h}$ the discrete finite volume solution, $\pi_{h} u$ the $L^{2}$ projection of $u$ on $\mathcal{P}_{0}\left(T_{h}\right)$ and $\varepsilon_{h}=u_{h}-\pi_{h} u$.

$$
\mathcal{L}_{h}\left(u_{h}, g\right)-\mathcal{L}_{h}\left(\pi_{h} u, g\right)=f_{h}-\mathcal{L}_{h}\left(\pi_{h} u, g\right)=\pi_{h}(\mathcal{L} u)-\mathcal{L}_{h}\left(\pi_{h} u, g\right) .
$$

Noticing that

$$
\begin{aligned}
\left(\pi_{h}(\mathcal{L} u)-\mathcal{L}_{h}\left(\pi_{h} u, g\right)\right)_{\mid K} & =\frac{1}{m(K)} \int_{K}\left(\mathcal{L} u-\mathcal{L}_{h}\left(\pi_{h} u, g\right)\right) \\
& =\frac{1}{m(K)} \sum_{e \in \partial K} s_{K, e} R_{e}(u) m(e)
\end{aligned}
$$

and using the linearity of $\mathcal{L}_{h}$ in $(30)$, we find

$$
\mathcal{L}_{h}\left(\varepsilon_{h}, 0\right)_{\mid K}=\frac{1}{m(K)} \sum_{e \in \partial K} s_{K, e} R_{e}(u) m(e) .
$$

Multiplying this equality by $\varepsilon_{K}$ and summing over all the cells, we obtain

$$
\left(\mathcal{L}_{h}\left(\varepsilon_{h}, 0\right), \varepsilon_{h}\right)_{L^{2}} \leq \sum_{K \in T_{h}} \varepsilon_{K} \sum_{e \in \partial K} s_{K, e} R_{e} m(e)=\sum_{e \in S_{h}} R_{e} \frac{\varepsilon_{E}-\varepsilon_{W}}{h_{e}} 2 m\left(\chi_{e}\right) .
$$

Afterwards, applying a Cauchy-Schwarz inequality for the right hand side:

$$
\left(\mathcal{L}_{h}\left(\varepsilon_{h}, 0\right), \varepsilon_{h}\right)_{L^{2}} \leq 2\left\|R_{h}\right\|_{L^{2}}\left\|\varepsilon_{h}\right\|_{1,0}
$$

Furthermore, using the hypotheses (12) to (14) and the results of Lemma 3.1 and Lemma 4.1 yields

$$
\left\|\varepsilon_{h}\right\|_{1,0} \leq C\left\|R_{h}\right\|_{L^{2}} \leq C h, \quad\left\|\varepsilon_{h}\right\|_{L^{2}} \leq C\left\|\varepsilon_{h}\right\|_{1,0} \leq C h .
$$

Finally, using $\left\|u-\pi_{h} u\right\|_{L^{2}} \leq C h$ completes the proof of the theorem. 


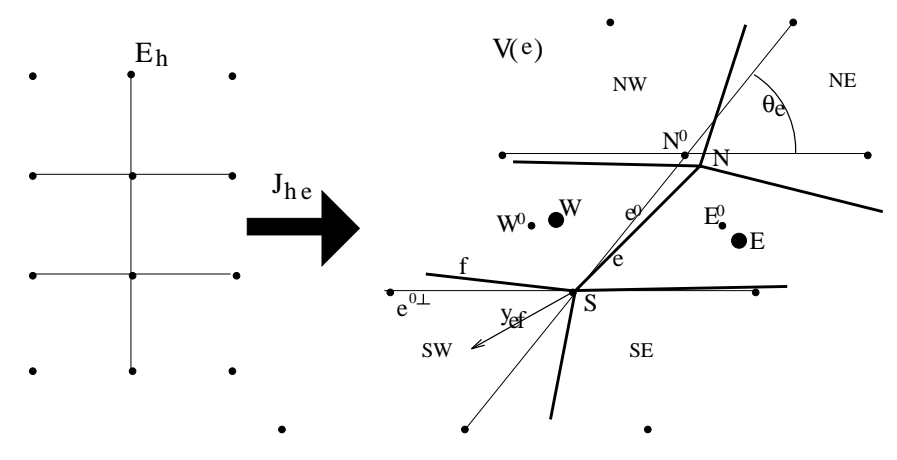

FiguRE 6.1.

\section{Convergence of the Diamond CELl Method on QuAdrangular meshes}

\subsection{Hypotheses on the mesh}

Let $E_{h}$ be a Cartesian grid made of the vertices $M_{i j}=(i h, j h)_{i=-1 \ldots 2 ; j=-1 \ldots 1}$ of six squares of length $h$ (see Fig. 6.1) and $Q_{h}=[-h, 2 h] \times[-h, h]$.

Definition 6.1. The family of meshes $\left(T_{h}\right)_{h>0}$ is said to be admissible if there exists a constant $B>0$ such that $\forall h>0, \forall e \in S_{h}^{\star}$ there exists $J_{h, e} \in C^{2}\left(Q_{h}, \mathbb{R}^{2}\right)$ such that

$$
\begin{gathered}
J_{h, e}\left(E_{h}\right)=\mathcal{V}(e), \\
\sup _{x \in Q_{h}}\left|\nabla J_{h, e}(x)\right|_{2} \leq B, \\
\sup _{x \in Q_{h}}\left|\nabla^{2} J_{h, e}(x)\right|_{2} \leq B,
\end{gathered}
$$

where $\mathcal{V}(e)$ denotes the set of the twelve vertices surrounding the edge $e$ of $S_{h}^{\star}$ ( $E_{h}$ may be truncated conveniently if $\bar{e} \cap \Gamma$ is not empty). $|\cdot|_{2}$ denotes the Euclidian norm.

Remark. If $J_{h, e}$ is linear $\left(\nabla J_{h, e}\right.$ is constant and $\left.\nabla^{2} J_{h, e}=0\right)$ then $J_{h, e}\left(Q_{h}\right)$ is a mesh of six parallelograms. Otherwise, let be $L J_{h, e}(x)=J_{h, e}\left(x_{0}\right)+\nabla J_{h, e}\left(x_{0}\right)$. $\left(x-x_{0}\right)$ the linear tangent application in $x_{0}$. The difference between $J_{h, e}\left(Q_{h}\right)$ (the real mesh) and $L J_{h, e}\left(Q_{h}\right)$ (a mesh of parallelogram) is bounded by the derivatives of $J_{h, e}$ and the characteristic size of the mesh $(h)$. Such meshes are easily proved to verify hypotheses (2).

\subsection{Main result}

Theorem 6.1. If $\left(T_{h}\right)_{h>0}$ is an admissible family of quadrangular meshes (Def. 6.1), then the finite volume scheme (10), associated with the weights (21), converges to the solution u of (1), and for $h$ small enough,

$$
\begin{aligned}
& \text { the discrete problem (10) is well-posed, } \\
& \qquad C C>0, \forall h>0, \quad\left\|u_{h}-\pi_{h} u\right\|_{1,0}+\left\|u_{h}-u\right\|_{L^{2}}<C h .
\end{aligned}
$$

Proof. Using Theorem 5.1, it just remains to prove the coercivity of the discrete operator $\mathcal{L}_{h}$. Let us first notice that, on the edges $e$ of $S_{h}^{\star}, u_{N}-u_{S}$ can be naturally spread out into some contributions $u_{E}-u_{W}$ on all the edges $f$ of $\mathcal{V}_{e}=\mathcal{V}_{N} \cup \mathcal{V}_{S}$ as follows. 
- If $\bar{e}$ does not intersect the boundary $\Gamma$, a short calculation yields

$$
\begin{aligned}
u_{N}-u_{S}= & y_{N W}(N)\left(u_{N W}-u_{W}\right)+y_{N E}(N)\left(u_{N E}-u_{E}\right) \\
& +y_{S W}(S)\left(u_{W}-u_{S W}\right)+y_{S E}(S)\left(u_{E}-u_{S E}\right) \\
& +\left(\left(y_{N E}(N)+y_{E}(N)\right)-\left(y_{E}(S)+y_{S E}(S)\right)\right)\left(u_{E}-u_{W}\right) .
\end{aligned}
$$

- If $\bar{e}$ does intersect the boundary $\Gamma$ with $N=\bar{e} \cap \Gamma$, using $u_{N}=0$, it is found

$$
\begin{aligned}
u_{N}-u_{S}= & -\left(y_{S W}(S)+y_{W}(S)\right) u_{W}-\left(y_{S E}(S)+y_{E}(S)\right) u_{E} \\
& +y_{S W}(S)\left(u_{W}-u_{S W}\right)+y_{S E}(S)\left(u_{E}-u_{S E}\right) \\
= & \frac{1}{2}\left(0-u_{W}\right)+\frac{1}{2}\left(0-u_{E}\right) \\
& +y_{S W}(S)\left(u_{W}-u_{S W}\right)+y_{S E}(S)\left(u_{E}-u_{S E}\right) \\
& +\left(\left(\frac{1}{2}+0\right)-\left(y_{E}(S)+y_{S E}(S)\right)\right)\left(u_{E}-u_{W}\right) .
\end{aligned}
$$

So, writing the previous general form prevails with the following notations:

$$
\begin{aligned}
y_{N W}(N)=\frac{1}{2}, y_{N E}(N) & =\frac{1}{2}, y_{W}(N)=0, y_{E}(N)=0, \\
u_{N W} & =u_{N E}=0 .
\end{aligned}
$$

- If $e$ does intersect the boundary $\Gamma$ with $\bar{e} \cap \Gamma=S$, the same expression remains, just swapping $N$ and $S$.

Let $\mathcal{P}_{e}$ be the set of all the edges $f$ perpendicular to $e$. The associated weight to such an edge $f \in \mathcal{P}_{e}$ is denoted by $y_{e f}$ :

$$
y_{e f}=y_{K}(P) \text { where } P=\bar{e} \cap \bar{f}, e \notin \partial K \text { while } f \in \partial K
$$

As a result, we have

where

$$
\forall e \in S_{h}^{\star} \quad u_{N}-u_{S}=\Delta u_{N S}^{0}+\delta y_{e}\left(u_{E}-u_{W}\right)_{e}
$$

$$
\begin{aligned}
\Delta u_{N S}^{0} & =\sum_{f \in \mathcal{P}_{e}} y_{e f}\left(u_{E}-u_{W}\right)_{f}, \\
\delta y_{e} & =\left(y_{N W}(N)+y_{W}(N)\right)-\left(y_{S W}(S)+y_{W}(S)\right) .
\end{aligned}
$$

At this stage, we note that, with the notations introduced above, $\mathcal{Q}_{h}^{D}\left(u_{h}\right)$ may be written as

$$
\forall u_{h} \in \mathcal{P}_{0}\left(T_{h}\right) \quad \frac{1}{2} \mathcal{Q}^{D}\left(u_{h}\right)=\left(A \mathbf{p}_{h}^{\perp}, \mathbf{p}_{h}\right)_{L^{2}}=\mathcal{Q}_{0}^{D}\left(u_{h}\right)+\delta \mathcal{Q}^{D}\left(u_{h}\right),
$$

where

$$
\begin{aligned}
\mathcal{Q}_{0}^{D}\left(u_{h}\right) & =\sum_{e \in S_{h}}\left(\frac{u_{E}-u_{W}}{h_{e}}+\frac{\beta_{e}}{\lambda_{e}} \frac{\Delta u_{N S}^{0}}{m(e)}\right) \frac{u_{E}-u_{W}}{h_{e}} \lambda_{e} m\left(\chi_{e}\right), \\
\delta \mathcal{Q}^{D}\left(u_{h}\right) & =\sum_{e \in S_{h}^{\star}} \frac{\beta_{e}}{\lambda_{e}} \delta y_{e}\left(\frac{u_{E}-u_{W}}{h_{e}}\right)^{2} \lambda_{e} m\left(\chi_{e}\right) .
\end{aligned}
$$

Afterwards the proof of coercivity is achieved by using the ideas of Lemma 4.1. The constant $\gamma$ of condition (25) is performed by collecting on each edge $f \in S_{h}$ the contributions issued of the outspreading of $\Delta u_{N S}^{0}$ [see (37)]. It is proved the following estimate (the definition of $\sigma_{e}$ will be precised in the proof). 
Lemma 6.1.

$$
\begin{aligned}
\sum_{e \in S_{h}^{\star}}\left(\frac{\beta_{e}}{\lambda_{e}}\right)^{2}\left(\frac{\Delta u_{N S}^{0}}{m(e)}\right)^{2} \lambda_{e} m\left(\chi_{e}\right) & \leq \sum_{f \in S_{h}} \gamma_{f}\left(\frac{\left(u_{E}-u_{W}\right)_{f}}{h_{f}}\right)^{2} \lambda_{f} m\left(\chi_{f}\right), \\
\forall f \in S_{h}, \gamma_{f} & =\sum_{e \in \mathcal{P}_{f} \cap S_{h}^{\star}} \sigma_{e} y_{e f}\left(\frac{\beta_{e}}{\lambda_{e}}\right)^{2} \frac{\lambda_{e}}{\lambda_{f}} \frac{h_{e}}{m(f)}
\end{aligned}
$$

Proof of Lemma 6.1.

$$
\frac{\beta_{e}}{\lambda_{e}} \frac{\Delta u_{N S}^{0}}{m(e)}=\frac{\beta_{e}}{\lambda_{e}} \sum_{f \in \mathcal{P}_{e}} y_{e f} \frac{h_{f}}{m(e)} \frac{\left(u_{E}-u_{W}\right)_{f}}{h_{f}} .
$$

Using Jensen inequality, we obtain

$$
\left(\frac{\beta_{e}}{\lambda_{e}}\right)^{2}\left(\frac{\Delta u_{N S}^{0}}{m(e)}\right)^{2} \leq\left(\frac{\beta_{e}}{\lambda_{e}}\right)^{2} \sigma_{e} \sum_{f \in \mathcal{P}_{e}} y_{e f} \frac{h_{f}}{m(e)}\left(\frac{\left(u_{E}-u_{W}\right)_{f}}{h_{f}}\right)^{2}
$$

where

$$
\sigma_{e}=\sum_{f \in \mathcal{P}_{e}} y_{e f} \frac{h_{f}}{m(e)} \quad\left(\text { for } e \in S_{h}^{\star}\right)
$$

Thus, we have

$$
\sum_{e \in S_{h}^{\star}}\left(\frac{\beta_{e}}{\lambda_{e}}\right)^{2}\left(\frac{\Delta u_{N S}^{0}}{m(e)}\right)^{2} \lambda_{e} m\left(\chi_{e}\right) \leq \sum_{e \in S_{h}^{\star}}\left(\frac{\beta_{e}}{\lambda_{e}}\right)^{2} \sigma_{e} \sum_{f \in \mathcal{P}_{e}} y_{e f} \frac{h_{f}}{m(e)}\left(\frac{\left(u_{E}-u_{W}\right)_{f}}{h_{f}}\right)^{2} \lambda_{e} m\left(\chi_{e}\right) .
$$

The desired result is achieved by swapping the two sums:

$$
\begin{aligned}
\sum_{e \in S_{h}^{\star}}\left(\frac{\beta_{e}}{\lambda_{e}}\right)^{2}\left(\frac{\Delta u_{N S}^{0}}{m(e)}\right)^{2} \lambda_{e} m\left(\chi_{e}\right) & \leq \sum_{f \in S_{h}} \gamma_{f}\left(\frac{\left(u_{E}-u_{W}\right)_{f}}{h_{f}}\right)^{2} \lambda_{f} m\left(\chi_{f}\right), \\
\gamma_{f} & =\sum_{e \in S_{h}^{\star} / f \in \mathcal{P}_{e}}\left(\frac{\beta_{e}}{\lambda_{e}}\right)^{2} \sigma_{e} y_{e f} \frac{h_{f}}{m(e)} \frac{\lambda_{e} m\left(\chi_{e}\right)}{\lambda_{f} m\left(\chi_{f}\right)} .
\end{aligned}
$$

Noticing that $\left\{e \in S_{h}^{\star}\right.$ such that $\left.f \in \mathcal{P}_{e}\right\} \Leftrightarrow e \in \mathcal{P}_{f} \cap S_{h}^{\star}, \gamma_{f}$ is simplified to be finally written as

$$
\gamma_{f}=\sum_{e \in \mathcal{P}_{f} \cap S_{h}^{\star}} \sigma_{e} y_{e f}\left(\frac{\beta_{e}}{\lambda_{e}}\right)^{2} \frac{\lambda_{e}}{\lambda_{f}} \frac{h_{e}}{m(f)} \quad\left(\text { for } f \in S_{h}\right) .
$$

To complete the proof, the main difficulty is to check the condition $0 \leq \gamma_{f}<1$ for each edge $f \in S_{h}$. Until this point, we only used the fact that the mesh was structured and we didn't take into account the geometric hypothesis (32)... (34) of Definition 6.1, which ensure the mesh $T_{h}$ to be locally near from a mesh of parallelograms. This last property is the key ingredient of the following lemma. 


\section{Lemma 6.2.}

$$
\begin{array}{rlrl}
\forall P \in A_{h}^{\star}, \quad \forall K & \in \mathcal{V}_{P}, \quad y_{K}(P) & =\frac{1}{4}+O(h) . \\
\forall e & \in S_{h}, \quad \delta y_{e} & =O(h) . \\
\forall e & \in S_{h}, & \gamma_{e} & \leq(1+O(h))\left(\frac{\beta_{e^{0 \perp}}}{\lambda_{e^{0 \perp}}}\right)^{2} \frac{\lambda_{e^{0 \perp}}}{\lambda_{e^{0}}} \sin ^{2} \theta_{e} .
\end{array}
$$

Remark. It can be easily verified that, on a mesh of parallelograms, $e$ and $e^{\perp}$ denoting the two directions of the edges and $\theta_{e}$ the measure of their angle, we have exactly:

$$
\begin{aligned}
& \forall P \in A_{h}^{\star}, \quad \forall K \in \mathcal{V}_{P}, \quad y_{K}(P)=\frac{1}{4}, \\
& \forall e \in S_{h}, \quad \delta y_{e}=0, \\
& \forall e \in S_{h}, \quad \gamma_{e}=\left(\frac{\beta_{e^{\perp}}}{\lambda_{e^{\perp}}}\right)^{2} \frac{\lambda_{e^{\perp}}}{\lambda_{e}} \sin ^{2} \theta_{e} .
\end{aligned}
$$

Hence, Lemma 6.2 is just a consequence of some Taylor expansions.

Proof of Lemma 6.2. We shall need a few notations. For any $h>0$, and any given edge $e \in S_{h}^{\star}$, let

- $J$ be the local $C^{2}$-bounded transformation from $E_{h}$ to $\mathcal{V}(e)$,

- $\xi_{i}$ be the vertices of $E_{h}$ [with $\left.\xi_{0}=(0,0)\right]$,

- $x_{i}=J\left(\xi_{i}\right)$ be the vertices of the mesh $T_{h}$,

- $x_{i}^{0}=x_{0}+\nabla J\left(\xi_{0}\right) \cdot \xi_{i}$ be the vertices of a new local mesh $\mathcal{V}^{0}(e)$ around $e$.

Using a Taylor expansion, it is found

$$
\begin{aligned}
& x_{i}=x_{i}^{0}+\int_{0}^{1} \xi_{i} \cdot \nabla^{2} J\left(\xi_{0}+\theta \xi_{i}\right) \cdot \xi_{i}(1-\theta) \mathrm{d} \theta, \\
& \left\|x_{i}-x_{i}^{0}\right\| \leq C h^{2} .
\end{aligned}
$$

This inequality shows that the error made when considering the mesh as made of parallelograms is of order $h^{2}$ on the vertices coordinates. Then all the following estimates are resulting from $\mathcal{V}^{0}(e)$ being made of parallelograms (see Fig. 6.1).

Let $K$ be a cell of $T_{h} . K$ is assumed to be defined by the vertices $x_{0} \ldots x_{3}$, while $K^{0}$ denotes the parallelogram $x_{0}^{0} \ldots x_{3}^{0}$. Let $G$ be the function which associates four non aligned points $\left(y_{0} \ldots y_{3}\right)$ of $\bar{\Omega}$ with the centre of gravity $y_{G}$ of the corresponding quadrangle. $G$ may be easily proved to be of class $C^{\infty}(\bar{\Omega})$, and consequently,

$$
\left\|x_{K}-x_{K}^{0}\right\| \leq C \sum_{i=0}^{3}\left\|x_{i}-x_{i}^{0}\right\| \leq C h^{2} .
$$

Let $P \in A_{h}^{\star}$. $P$ can be defined as $x_{0}=J\left(\xi_{0}\right)$ for an edge $e \in S_{h}^{\star}$. Besides the four cells $K \in \mathcal{V}_{P}$ have their vertices in $\mathcal{V}(e)=J\left(E_{h}\right)$, and $x_{0}$ can be supposed to be the origin $\left(x_{0}=(0,0)\right)$. Hence, using (41), their centres $x_{K}$ verify

$$
\forall K \in \mathcal{V}_{P}, x_{K}=x_{K}^{0}+O\left(h^{2}\right)=\nabla J\left(\xi_{0}\right) \cdot \xi_{K}+O\left(h^{2}\right) .
$$

But for the mesh of parallelograms $\mathcal{V}^{0}(e)$, we obtain

$$
R_{x}^{0}=\sum_{\mathcal{V}_{P}^{0}} x_{K}^{0}=\nabla J\left(\xi_{0}\right) \cdot \sum_{\mathcal{V}_{P}^{0}} \xi_{K}=0, \quad R_{y}^{0}=\sum_{\mathcal{V}_{P}^{0}} y_{K}^{0}=0
$$


and consequently,

$$
R_{x}=O\left(h^{2}\right), \quad R_{y}=O\left(h^{2}\right) .
$$

Besides using that $\nabla J$ is uniformly bounded, we have

$$
x_{K}=O(h), \quad y_{K}=O(h), \quad I_{x x}=O\left(h^{2}\right), \quad I_{y y}=O\left(h^{2}\right), \quad I_{x y}=O\left(h^{2}\right),
$$

thus,

$$
D=O\left(h^{4}\right), \quad \lambda_{x}=O(1), \quad \lambda_{y}=O(1)
$$

Finally it yields

$$
y_{K}(P)=\frac{1}{4}+O(h)
$$

Let $f \in S_{h}$. The endpoint $S$ of the edge $f$ is supposed to be $x_{0}=J\left(\xi_{0}\right)$. For any edge $e \in \mathcal{V}(f)$ supposed to be of endpoints $x_{1}$ and $x_{2}, e^{0}$ denotes the edge $x_{1}^{0} x_{2}^{0}$ in the mesh $T_{h}^{0}$ of parallelograms.

$$
\left|m(e)-m\left(e^{0}\right)\right|=\left|\left\|x_{1}-x_{2}\right\|-\left\|x_{1}^{0}-x_{2}^{0}\right\|\right| \leq\left\|x_{1}-x_{1}^{0}\right\|+\left\|x_{2}-x_{2}^{0}\right\| \leq C h^{2} .
$$

Let $E$ and $W$ be the centres of the two neighbours of a given edge $e \in \mathcal{V}(f)$ and $E^{0}$ and $W^{0}$ the corresponding centres in the local mesh $T_{h}^{0}$ of parallelograms. The following estimates are straightforward.

$$
\begin{aligned}
\left|h_{e}-h_{e^{0}}\right| & =\left|\left(x_{W}-x_{E}\right) \cdot \mathbf{n}_{e}-\left(x_{W}^{0}-x_{E}^{0}\right) \cdot \mathbf{n}_{e^{0}}\right| \\
& =\left|\frac{\left\|\left(x_{W}-x_{E}\right) \wedge\left(x_{N}-x_{S}\right)\right\|}{\left\|x_{N}-x_{S}\right\|}-\frac{\left\|\left(x_{W}^{0}-x_{E}^{0}\right) \wedge\left(x_{N}^{0}-x_{S}^{0}\right)\right\|}{\left\|x_{N}^{0}-x_{S}^{0}\right\|}\right| \\
& \leq C h^{2} . \\
\left|\alpha_{e}-\alpha_{e^{0}}\right| & =\left|\frac{\left(x_{W}-x_{E}\right) \cdot\left(x_{N}-x_{S}\right)}{h_{e}\left\|x_{N}-x_{S}\right\|}-\frac{\left(x_{W}^{0}-x_{E}^{0}\right) \cdot\left(x_{N}^{0}-x_{S}^{0}\right)}{h_{e^{0}}\left\|x_{N}^{0}-x_{S}^{0}\right\|}\right| \leq C h .
\end{aligned}
$$

Besides, the following properties are clearly observed on the mesh $T_{h}^{0}$.

$$
\begin{gathered}
\text { If } e \in \mathcal{P}_{f} \cap S_{h}^{\star}, \quad \frac{h_{e^{0}}}{m\left(f^{0}\right)}=\left|\sin \theta_{f}\right| . \\
\text { If } f \in S_{h}^{\star}, e \in \mathcal{P}_{f}, \quad \frac{h_{e^{0}}}{m\left(f^{0}\right)}=\left\{\begin{array}{c}
\left|\sin \theta_{f}\right|\left(e \in S_{h}^{\star}\right) \\
\frac{1}{2}\left|\sin \theta_{f}\right|\left(e \in \Gamma_{h}\right),
\end{array}\right.
\end{gathered}
$$

where $\theta_{f}$ denotes the angle of the parallelograms in the mesh $T_{h}^{0}$.

Calculating the matrix $A_{e}$ in the basis $\left(\mathbf{n}_{e^{0}}, \mathbf{t}_{e^{0}}\right)$ for $e \in \mathcal{V}(f)$ proceeds by evaluating the formulae of changing bases

$$
A_{e^{0}}=\left[\begin{array}{cc}
\cos \eta & -\sin \eta \\
\sin \eta & \cos \eta
\end{array}\right] A_{e}\left[\begin{array}{cc}
\cos \eta & \sin \eta \\
-\sin \eta & \cos \eta
\end{array}\right]
$$


where $\eta$ is the angle between the exact edge $e$ and the approximate one $e^{0}$; furthermore,

$$
\begin{aligned}
|\cos \eta| & =\frac{\left|\left(x_{1}-x_{2}\right) \cdot\left(x_{1}^{0}-x_{2}^{0}\right)\right|}{m(e) m\left(e^{0}\right)} \\
& =\frac{\left\|x_{1}^{0}-x_{2}^{0}\right\|^{2}}{m(e)^{2}(1+O(h))}+O(h)=1+O(h), \\
|\sin \eta| & =\frac{\left\|\left(x_{1}-x_{2}\right) \wedge\left(x_{1}^{0}-x_{2}^{0}\right)\right\|}{m(e) m\left(e^{0}\right)} \\
& =\frac{\left\|\left(\left(x_{1}-x_{2}\right)-\left(x_{1}^{0}-x_{2}^{0}\right)\right) \wedge\left(x_{1}^{0}-x_{2}^{0}\right)\right\|}{m(e) m\left(e^{0}\right)}=O(h) .
\end{aligned}
$$

At last, for $f \in S_{h}$ and $e \in \mathcal{P}_{f} \cap S_{h}^{\star}$, we have

$$
\begin{aligned}
& \frac{\beta_{e}}{\lambda_{e}}=\frac{\beta_{e^{0}}}{\lambda_{e^{0}}}(1+O(h))=\frac{\beta_{f^{0 \perp}}}{\lambda_{f^{0 \perp}}}(1+O(h)), \\
& \frac{\lambda_{e}}{\lambda_{f}}=\frac{\lambda_{e^{0}}}{\lambda_{f^{0}}}(1+O(h))=\frac{\lambda_{f^{0 \perp}}}{\lambda_{f^{0}}}(1+O(h)),
\end{aligned}
$$

where $f^{0 \perp}$ denotes the constant direction of the edges $e^{0}$ for $e \in \mathcal{P}_{f}$.

The evaluation of $\gamma_{e}$ and $\delta y_{e}$ proceeds now by collecting all the previous estimates. $\forall e \in S_{h}^{\star}$,

$$
\begin{aligned}
\sigma_{e} & =\sum_{f \in \mathcal{P}_{e}} y_{e f} \frac{h_{f}}{m(e)}=\left(\sum_{\mathcal{P}_{e} \cap S_{h}^{\star}} \frac{1}{4}\left|\sin \theta_{e}\right|+\sum_{\mathcal{P}_{e} \cap \Gamma_{h}} \frac{1}{2} \frac{1}{2}\left|\sin \theta_{e}\right|\right)(1+O(h)) \\
& =\left|\sin \theta_{e}\right|(1+O(h)) .
\end{aligned}
$$

The final estimate of $\gamma_{e}$ derives itself from the next remark on the local angles of the parallelograms on two neighbouring edges.

$$
\begin{array}{rlrl}
\forall e, f \in S_{h} / \bar{e} \cap \bar{f} \neq \emptyset, & \left.\left|\theta_{e}\right|=\left|\left(\widehat{e^{0}, e^{0 \perp}}\right)\right|=\mid \widehat{(e, f}\right) \mid(1+O(h)), \\
\left|\theta_{f}\right|=\left|\left(\widehat{f^{0}, f^{0 \perp}}\right)\right|=|(\widehat{f, e})|(1+O(h)),
\end{array}
$$

and so,

$$
\forall e \in S_{h}^{\star}, \quad \forall f \in \mathcal{P}_{e}, \quad\left|\theta_{e}\right|=\left|\theta_{f}\right|(1+O(h)) \quad \text { and } \quad \sigma_{e}=\left|\sin \theta_{f}\right|(1+O(h)) .
$$

Afterwards, $\gamma_{f}$ and $\delta y_{e}$ are performed easily:

$$
\begin{aligned}
& \forall e \in S_{h}^{\star}, \quad \delta y_{e}=O(h), \\
& \forall f \in S_{h}, \quad \gamma_{f} \leq\left(\frac{\beta_{f^{0 \perp}}}{\lambda_{f^{0 \perp}}}\right)^{2} \frac{\lambda_{f^{0 \perp}}}{\lambda_{f^{0}}} \sin ^{2} \theta_{f}(1+O(h)) .
\end{aligned}
$$

End of the proof of Theorem 6.1. The coercivity is achieved by substituting $(42,43)$ in the expression of $\delta \mathcal{Q}^{D}$ and in Lemma 4.1. The remainder $\delta \mathcal{Q}^{D}$ is obviously estimated.

$$
\left|\delta \mathcal{Q}^{D}\right| \leq \sum_{e \in S_{h}^{\star}}\left|\beta_{e} \delta y_{e}\right|\left(\frac{u_{E}-u_{W}}{h_{e}}\right)^{2} m\left(\chi_{e}\right) \leq \beta_{\max } O(h)\left\|u_{h}\right\|_{1,0}^{2}
$$

(since $\beta_{e}=\mu_{e}-\alpha_{e} \lambda_{e}$ is clearly uniformly bounded). 
Furthermore, Lemma 4.1 provides the following estimate of $\mathcal{Q}_{0}^{D}$.

$$
\mathcal{Q}_{0}^{D}\left(u_{h}\right) \geq 2 \inf _{h>0, e \in S_{h}} \frac{\lambda_{e}\left(1-\gamma_{e}\right)}{2}\left\|u_{h}\right\|_{1,0}^{2} .
$$

Finally a more precise estimate of $\lambda_{e}\left(1-\gamma_{e}\right)$ brings out the final constant of coercivity.

$$
\begin{aligned}
\lambda_{e}\left(1-\gamma_{e}\right) & \geq \lambda_{e^{0}}\left(1-\left(\frac{\beta_{e^{0 \perp}}}{\lambda_{e^{0 \perp}}}\right)^{2} \frac{\lambda_{e^{0 \perp}}}{\lambda_{e^{0}}} \sin ^{2} \theta_{e}\right)(1+O(h)) \\
& =\left(\lambda_{e^{0}} \lambda_{e^{0 \perp}}-\beta_{e^{0 \perp}}^{2} \sin ^{2} \theta_{e}\right) \frac{1}{\lambda_{e^{0 \perp}}}(1+O(h)) \\
& =\left(\lambda_{e^{0}} \lambda_{e^{0 \perp}}-\left(\mu_{e^{0 \perp}} \sin \theta_{e}+\lambda_{e^{0 \perp}} \cos \theta_{e}\right)^{2}\right) \frac{1}{\lambda_{e^{0 \perp}}}(1+O(h)),
\end{aligned}
$$

because $\beta_{e^{0 \perp}}=\mu_{e^{0 \perp}}-\alpha_{e^{0 \perp}} \lambda_{e^{0 \perp}}$ and $\alpha_{e^{0 \perp}}=-\operatorname{cotan} \theta_{e}$ (see Fig. 6.1).

Using the previous changing bases formulae from the basis of $e^{0 \perp}$ to the basis of $e^{0}$, between which the angle is actually $\theta_{e}$ yields

$$
\lambda_{e^{0}}=\lambda_{e^{0 \perp}} \cos ^{2} \theta_{e}+\nu_{e^{0 \perp}} \sin ^{2} \theta_{e}+2 \mu_{e^{0 \perp}} \cos \theta_{e} \sin \theta_{e}
$$

and consequently,

$$
\begin{aligned}
\lambda_{e}\left(1-\gamma_{e}\right) & =\left(\nu_{e^{0 \perp}} \lambda_{e^{0 \perp}}-\mu_{e^{0 \perp}}^{2}\right) \sin ^{2} \theta_{e} \frac{1}{\lambda_{e^{0 \perp}}}(1+O(h)) \\
& =\frac{\operatorname{det} A_{e^{0 \perp}} \sin ^{2} \theta_{e}}{\lambda_{e^{0 \perp}}}(1+O(h)) \\
& \geq \lambda_{\min } \sin ^{2} \theta_{\min }(1+O(h)),
\end{aligned}
$$

where $\theta_{\min }$ denotes the smallest angle in the cells $K$ of the $\left(T_{h}\right)_{h>0}$. This completes the proof.

\section{REFERENCES}

[1] R.A. Adams, Sobolev Spaces. Academic Press, New-York (1975).

[2] M. Aftosmis, D. Gaitonde and T. Sean Tavares, On the accuracy, stability and monotonicity of various reconstruction algorithms for unstructured meshes. AIAA paper No. 94-0415 (1994).

[3] J. Baranger, J.F. Maitre and F. Oudin, Connection between finite volume and mixed finite element methods. RAIRO Modél. Math. Anal. Num. 30 (1996) 445-465.

[4] W.J. Coirier, An Adaptatively-Refined, Cartesian, Cell-based Scheme for the Euler and Navier-Stokes Equations. Ph.D. thesis, Michigan University, NASA Lewis Research Center, USA (1994).

[5] W.J. Coirier and K.G. Powell, A Cartesian, cell-based approach for adaptative-refined solutions of the Euler and Navier-Stokes equations. AIAA (1995)

[6] Y. Coudière, Analyse de schémas volumes finis sur maillages non structurés pour des problèmes lináires hyperboliques et elliptiques. Ph.D. thesis, Paul Sabatier University, France (1998).

[7] M. Dauge, Elliptic Boundary Value Problems in Corner Domains. Lect. Notes Math. 1341, Springer Verlag, Berlin-New York (1988).

[8] F. Dubois, Interpolation de Lagrange et volumes finis. Une technique nouvelle pour calculer le gradient d'une fonction sur les faces d'un maillage non structuré. Technical report, Aérospatiale (1992).

[9] I. Faille, A control volume method to solve an elliptic equation on a 2d irregular meshing. Comput. Methods. Appl. Mech. Engrg. 100 (1992) 275-290.

[10] T. Gallouët, An introduction to finite volume methods. Technical report, Cours CEA/EDF/INRIA (1992).

[11] W. Guo and M. Stynes, An analysis of a cell-vertex finite volume method for a parabolic convection-diffusion problem. Math. Comp. 66 (1997) 105-124.

[12] R. Herbin, An error estimate for a finite volume scheme for a diffusion-convection problem on a triangular mesh. Numerical Method for Partial Differential Equations 11 (1994) 165-173. 
[13] R. Herbin, Finite volume methods for diffusion convection equations on general meshes, in Finite Volumes for Complex Applications, Hermès (1996) 153-160.

[14] F. Jacon and D. Knight, A Navier-Stokes algorithm for turbulent flows using an unstructured grid and flux difference splitting. AIAA paper No. 94-2292 (1994).

[15] C.R. Mitchell and R.W. Walters, K-exect reconstruction for the Navier-Stokes equations on arbitrary grids. AIAA (1993).

[16] K.W. Morton and E. Süli, Finite volume methods and their analysis. IMA J. Numer. Anal. 11 (1991) 241-260.

[17] E. Süli, Convergence of finite volume schemes for Poisson's equation on nonuniform meshes. SIAM J. Numer. Anal. 28 (1991) $1419-1430$.

[18] J.-M. Thomas and D. Trujillo, Analysis of finite volumes methods. Technical Report 95/19, CNRS, URA 1204 (1995).

[19] J.-M. Thomas and D. Trujillo, Convergence of finite volumes methods. Technical Report 95/20, CNRS, URA 1204 (1995).

[20] D. Trujillo, Couplage espace-temps de schémas numériques en simulation de réservoir. Ph.D. thesis, University of Pau et pays de l'Adour (1994).

[21] S. Verdière and M.H. Vignal, Numerical and theoretical study of a dual mesh method using finite volume schemes for two phase flow problems in porous media. Numer. Math. 80 (1998) 601-639.

[22] P. Villedieu, Une méthode de volumes finis sur maillages non-structurés quelconques pour l'équation de convection diffusion. Technical Report 1-3550.00-DERI, ONERA (1996). 